\title{
Embeddings of propositional monomodal logics
}

\author{
Evgeni E. Zolin \\ Chair of Mathematical Logic \\ Faculty of Mathematics and Mechanics \\ Moscow State University, 119899 Moscow, Russia \\ e-mail: zolin@lpcs.math.msu.ru
}

\begin{abstract}
The aim of this paper is to investigate the expressibility of classical propositional monomodal logics. To this end, a notion of embedding of one logic into another is introduced, which is, roughly, a translation preserving theoremhood. This enables to measure the expressibility of a logic by a (finite or infinite) number of logics embeddable into it. This measure is calculated here for a large family of modal logics including K, K4, KB, K5, GL, T, S4, B, S5, Grz, and provability logics. It is also shown that some of these logics (e.g., all normal logics containing the symmetry axiom except for the logics Triv, Ver, and the intersection of these two) are not embeddable into some others (e.g., K, K4, K5, GL, T, S4, Grz).
\end{abstract}

Keywords: modal logic, provability logic, embedding, expressibility.

\section{Introduction}

In this paper we consider logics in the propositional language augmented by a unary modal operator $\square$. Let Fm denote the set of formulas of this language. Each formula $\varphi(p) \in \mathrm{Fm}$ of at most one variable $p$ induces a modality, i.e., an operator $\nabla_{\varphi}: \mathrm{Fm} \rightarrow$ Fm defined by $\nabla_{\varphi}(A):=\varphi(A)$, for all $A \in \mathrm{Fm}$. The familiar examples of modalities are the operators of necessity $\square$, possibility $\diamond$ (i.e., $\neg \square \neg$ ), and non-contingency $\Delta$ (induced by the formula $\square p \vee \square \neg p$ ).

Given a modal logic $L$, it is natural to measure its expressive power by a number of "distinct" modalities in $L$. However, there are (at least) two different approaches to formalise the quoted word.

According to the first, or internal, approach, modalities are identified if they are equivalent in $L$, i.e., if the equivalence of formulas they are induced by is a theorem of $L$. Typical results in the scope of this approach can be found in [3, p. 10], $[5,11,15,17]$, though in these papers only linear modalities, i.e. sequences of $\square \mathrm{s}$ and $\neg \mathrm{s}$, are mainly under consideration.

The second, or external, approach prescribes not to distinguish between modalities having an identical "behaviour" over $L$. Before giving a more precise description of this approach, let us consider an illustration. 
For $n>0$, denote by $\square^{n}$ a sequence of $n \square$ s. Modalities $\square^{n}$ are known to be pairwise non-equivalent in the logic $\mathbf{B}$ (see Subsection 3.2 for the definition of $\mathbf{B}$ ) and hence they are distinct from the viewpoint of the internal approach. However, no formula distinguishes them. That is, if we denote by $A^{n}$ the result of replacing of all $\square$ s in a formula $A$ by $\square^{n}$ then $A$ is a theorem of $\mathbf{B}$ iff $A^{n}$ is, as will be proved in Theorem 3.22. In this situation we can say that modalities $\square^{n}$ have the same behaviour over $\mathbf{B}$.

In general, to each modality $\nabla$ we assign a $\nabla$-translation $\operatorname{tr}_{\nabla}$ of formulas by putting $\operatorname{tr}_{\nabla}(A)$ to be the result of replacing of all occurrences of the symbol $\square$ in a formula $A$ by the operator $\nabla$. Further, we define a logic $L(\nabla)$ of a modality $\nabla$ over a logic $L$ as the set of all formulas whose $\nabla$-translations are theorems of $L$. Finally, a logic $M$ is called embeddable into $L$ if $M=L(\nabla)$ for some modality $\nabla$; here $\operatorname{tr}_{\nabla}$ serves as an embedding of $M$ into $L$ in the sense that, for all $A \in \mathrm{Fm}, A$ is a theorem of $M$ iff $\operatorname{tr}_{\nabla}(A)$ is a theorem of $L$. Thus, the external approach prescribes, given a logic $L$, to identify modalities having equal logics over $L$; we call these modalities analogous over $L$.

A rather close but different is the notion of simulation of modal logics explored in [8]; therein, a simulation is a translation of a more general kind and it is to preserve not only theoremhood but also a consequence relation.

Let us mention some well-known results and concepts related to the external approach. In [3, Chapter 12] the logic of a modality $\square$ (induced by $p \wedge \square p$ ) over the Gödel-Löb logic GL is proved to coincide with the Grzegorczyk logic Grz, i.e., $\mathbf{G L}(\bullet)=\mathbf{G r z}$. It is also shown there that GL is not embeddable into Grz. In the same way, one can easily see that $\mathbf{K}(\bullet)=\mathbf{T}$ and $\mathbf{K} \mathbf{4}(\bullet)=\mathbf{S 4}$, whereas $\mathbf{K}$ is not embeddable into $\mathbf{T}$, as well as $\mathbf{K} 4$ into $\mathbf{S} 4$. A logic $L$ is called iterative if $L\left(\square^{n}\right)=L$, for all $n>0$. In [1] this property was considered for the well-known family of provability logics (cf. $[2,7]$ ). In $[6,9]$ the logics of non-contingency modality $\Delta$ over $\mathbf{K}$ and $\mathbf{K} \mathbf{4}$ are axiomatised, whereas in $[12,13,14]$ the same is done for $\mathbf{T}, \mathbf{S 4}, \mathbf{S 5}$, and some other logics.

In this paper we address some issues within the framework of the external approach. The paper is organised as follows. Section 1 introduces basic notions and notation. In Section 2 a family of 15 logics of so called prime modalities is found and is proved to be exhaustive in the sense that a logic of any prime modality over any (consistent) logic belongs to the family. In Section 3 we measure the expressibility of the logics of prime modalities, some normal logics, and the provability logics. The iterativity of the logic B is also established in Subsection 3.3. The final Section 4 presents new positive and negative results concerning a possibility of embedding of some particular logics into some others. In particular, we show that any normal extension of the logic KB except for Triv, Ver, and Triv $\cap$ Ver is not embeddable into K, K4, K5, GL, T, S4, Grz, and some provability logics.

\section{Definitions and notation}

The propositional monomodal language consists of a denumerable set of variables Var $=\left\{p_{0}, p_{1}, \ldots\right\}$, symbols for falsehood $\perp$, implication $\rightarrow$, and a unary modal 
operator $\square$. Other connectives $(\top, \neg, \wedge, \vee, \leftrightarrow, \diamond)$ are taken as standard abbreviations. The set of formulas Fm is defined as usual.

Definition 1.1 A modality induced by a formula $\varphi(p)$ of at most one variable $p$ is an operator $\nabla: \mathrm{Fm} \rightarrow \mathrm{Fm}$ defined by $\nabla(A):=\varphi(A)$, for all $A \in \mathrm{Fm}$.

Modalities induced by formulas $\perp, \top, p, \neg p, p \wedge \square p$, and $\square^{n} p$, where $n>0$, will be denoted by $\perp, \top, \bigcirc, \neg, \square$, and $\square^{n}$, respectively. Clearly, all modalities are built up from $\perp$ and $\bigcirc$ using $\rightarrow$ and $\square$.

Definition 1.2 Given a modality $\nabla$, a $\nabla$-translation is a map $\operatorname{tr}_{\nabla}: \mathrm{Fm} \rightarrow \mathrm{Fm}$ defined as follows: $\operatorname{tr}_{\nabla}(\perp)=\perp ; \operatorname{tr}_{\nabla}(p)=p$, for any $p \in \operatorname{Var} ; \operatorname{tr}_{\nabla}(A \rightarrow B)=\operatorname{tr}_{\nabla}(A) \rightarrow \operatorname{tr}_{\nabla}(B)$; $\operatorname{tr}_{\nabla}(\square A)=\nabla\left(\operatorname{tr}_{\nabla}(A)\right)$. That is, $\operatorname{tr}_{\nabla}$ replaces all $\square$ s by $\nabla \mathrm{s}$.

Definition 1.3 A (classical propositional monomodal) logic is a set $L \subseteq$ Fm containing all classical tautologies in the modal language and closed under the rules of modus ponens and substitution:

$$
\text { (MP) } \frac{A \quad A \rightarrow B}{B} \quad \text { (Sub) } \frac{A}{A[B / p]}
$$

Here $A[B / p]$ denotes the result of simultaneous substituting a formula $B$ for all occurrences of a variable $p$ in $A$. In the sequel, we consider only consistent logics, i.e., proper subsets of Fm. We often use a notation $L \vdash A$ instead of $A \in L$. For $L \subseteq N \subseteq$ Fm, denote $[L, N]:=\{M \mid L \subseteq M \subseteq N\}$.

$\mathbf{E}$ denotes the smallest logic closed under the rule of equivalent replacement:

$$
\text { (RE) } \frac{A \leftrightarrow B}{\square A \leftrightarrow \square B}
$$

If $L$ is a calculus given by a set of axioms and rules and $X \subseteq$ Fm then denote by $L+X$ the calculus obtained from $L$ by adding formulas of $X$ as axiom schemata and by $L X$ the calculus whose axioms are theorems of $L$ and formulas of $X$ taken as axiom schemata and whose only rule is (MP).

Definition 1.4 A logic of a modality $\nabla$ over a logic $L$ is the set of all formulas whose $\nabla$-translations are theorems of $L$ :

$$
L(\nabla):=\left\{A \in \mathrm{Fm} \mid L \vdash \operatorname{tr}_{\nabla}(A)\right\}=\operatorname{tr}_{\nabla}^{-1}(L) .
$$

It is readily seen that $L(\nabla)$ is indeed a logic. Moreover, if $L$ is closed under the rule (RE) then so is $L(\nabla)$.

A logic $M$ is called embeddable into $L(M \hookrightarrow L$, in symbols) if $M=L(\nabla)$ for some modality $\nabla$.

Definition 1.5 Modalities $\nabla$ and $\Delta$ are called equivalent in $L$ if $L \vdash \nabla p \leftrightarrow \Delta p$; analogous over $L$ if $L(\nabla)=L(\Delta)$. 
If $L$ is closed under (RE) then any two modalities equivalent in $L$ are analogous over $L$, since $L \vdash \nabla p \leftrightarrow \Delta p$ implies $L \vdash \operatorname{tr}_{\nabla}(A) \leftrightarrow \operatorname{tr}_{\Delta}(A)$, for all $A \in \mathrm{Fm}$. The converse does not hold in general, as was already noted in Introduction: modalities $\square^{n}$ are non-equivalent but analogous over the logic $\mathbf{B}$.

Definition 1.6 A constant is a formula containing no variables; a modality induces by it will also be called a constant. A constant $\nabla$ is called trivial in a logic $L$ if either $L \vdash \nabla$ or $L \vdash \neg \nabla$; otherwise it is called proper in $L$.

A modality induced by a formula $\varphi(p)$ having no occurrences of $p$ in the scope of $\square$ is called prime. A prime logic is a logic of any prime modality over any (consistent) logic. Any prime logic is closed under (RE) even if $L$ is not.

\section{Prime logics}

In this section we show that there are exactly 15 prime logics and present natural axiomatisations thereof. We also show that, given a logic $L$ and a prime modality $\nabla$, to determine which of these 15 logics equals $L(\nabla)$ it suffices to find the characteristic function (c.f.) of $\nabla$ over $L$. We shall see that the latter problem is decidable whenever the variable-free fragment of $L$ is decidable. Therefore, a c.f. contains the full information about the behaviour of $\nabla$ over $L$.

Throughout, we identify a boolean function $f: \mathbf{2}^{n} \rightarrow \mathbf{2}$, where $\mathbf{2}=\{\perp, \top\}$, with any (fixed) boolean formula (i.e., formula containing no $\square$ s) representing $f$, e.g., with its full disjunctive normal form (FDNF):

$$
f(\vec{x})=\underset{\left\{\vec{\sigma} \in \mathbf{2}^{n} \mid f(\vec{\sigma})=\top\right\}}{\bigvee} \vec{p}^{\vec{\sigma}},
$$

where $\vec{x}=\left(x_{1}, \ldots, x_{n}\right), \vec{\sigma}=\left(\sigma_{1}, \ldots, \sigma_{n}\right), \vec{p}^{\vec{\sigma}}=p_{1}^{\sigma_{1}} \wedge \ldots \wedge p_{n}^{\sigma_{n}}, p^{\top}=p, p^{\perp}=\neg p$.

Definition 2.1 A characteristic function (c.f.) of a modality $\nabla$ over a logic $L$ is the least element of a set

$$
F_{\nabla}^{L}=\{f(x, y): \mathbf{2} \times \mathbf{2} \rightarrow \mathbf{2} \mid L \vdash f(\nabla \perp, \nabla \top)\}
$$

w.r.t. the following partial order:

$$
f \leqslant g \quad \Longleftrightarrow \quad \text { a formula } f \rightarrow g \text { is a tautology. }
$$

Each $\nabla$ has a unique c.f. since $F_{\nabla}^{L}$ is non-empty, finite, and closed under the pointwise conjunction of functions, hence its least element is merely the conjunction of all its elements.

Let $\nabla$ be a prime modality, then a formula $\nabla p$ is truth-functionally equivalent to $(p \wedge \nabla \top) \vee(\neg p \wedge \nabla \perp)$. Hence any prime logic contains a formula

$$
\square p \leftrightarrow[(p \wedge \square \top) \vee(\neg p \wedge \square \perp)] .
$$


Theorem 2.2 If $\chi_{i}$ is a c.f. of a modality $\nabla_{i}$ over a logic $L_{i}, i=1,2$, then

$$
L_{1}\left(\nabla_{1}\right) \subseteq L_{2}\left(\nabla_{2}\right) \quad \Longleftrightarrow \quad \chi_{2} \leqslant \chi_{1} .
$$

Proof. Let $F_{i}:=F_{\nabla_{i}}^{L_{i}}$ and $M_{i}:=L_{i}\left(\nabla_{i}\right)$. Clearly, $f \in F_{i} \Leftrightarrow f \geqslant \chi_{i}$. So $F_{1} \subseteq F_{2} \Leftrightarrow$ $\chi_{2} \leqslant \chi_{1}$. Since $M_{1} \subseteq M_{2}$ implies $F_{1} \subseteq F_{2}$, it remains to prove the converse.

Assume that $F_{1} \subseteq F_{2}$ and take any formula $A(\vec{p}) \in M_{1}$, where $\vec{p}=\left(p_{1}, \ldots, p_{n}\right)$ is the list of all variables in $A$. By ( $\downarrow), A$ is equivalent in $M_{1}$ to a boolean combination $b(\vec{p}, \square \perp, \square \top)$ of variables $\vec{p}$ and the constants $\square \perp$ and $\square \top$, whence $b(\vec{p}, \square \perp, \square \top) \in$ $M_{1}$. Then $b(\vec{\sigma}, \square \perp, \square \top) \in M_{1}$ and so $b(\vec{\sigma}, x, y) \in F_{1}$, for all $\vec{\sigma} \in \mathbf{2}^{n}$. But $F_{1} \subseteq F_{2}$, so $b(\vec{\sigma}, x, y) \in F_{2}$, for all $\vec{\sigma} \in \mathbf{2}^{n}$. The backward reasoning yields $b(\vec{p}, \square \perp, \square \top) \in M_{2}$ and finally $A(\vec{p}) \in M_{2}$, by $(\natural)$.

Corollary 2.3 Under the conditions of Theorem 2.2,

$$
L_{1}\left(\nabla_{1}\right)=L_{2}\left(\nabla_{2}\right) \quad \Longleftrightarrow \quad \chi_{1}=\chi_{2}
$$

Consequently, there are no more than 15 prime logics since each of them is determined by a binary boolean function $\chi \not \equiv \perp$. On the one hand, given a logic $L$, there exist at least 4 prime modalities having distinct logics over $L$, namely, $\perp, \bigcirc, \neg$, and $\top$. Moreover, the c.f. of each of them is independent of $L$ :

$$
\chi_{\nabla}(x, y)=x^{\nabla \perp} \wedge y^{\nabla \top}, \quad \nabla \in\{\perp, \bigcirc, \neg, \top\},
$$

and so are logics of these modalities. We denote these logics by $\boldsymbol{\Lambda}_{\perp}, \boldsymbol{\Lambda}_{\bigcirc}, \boldsymbol{\Lambda}_{\neg}$, and $\boldsymbol{\Lambda}_{\top}$ (the traditional names of $\boldsymbol{\Lambda}_{\bigcirc}$ and $\boldsymbol{\Lambda}_{\top}$ are Triv and Ver, respectively).

However, on the other hand, nothing guarantees the existence of modalities with other c.f.'s over a given $L$; for instance, in the above four logics any modality is equivalent to either $\perp, \bigcirc, \neg$, or $\top$. Nevertheless, Lemma 2.4 below argues that each $\chi \not \equiv \perp$ is "realisable."

Let $\varnothing \neq \Upsilon \subseteq\{\perp, \bigcirc, \neg, \top\}$ and put

$$
\boldsymbol{\Lambda}_{\Upsilon}:=\bigcap_{\nabla \in \Upsilon} \boldsymbol{\Lambda}_{\nabla} \quad \text { and } \quad \chi_{\Upsilon}(x, y):=\bigvee_{\nabla \in \Upsilon} \chi_{\nabla}(x, y)
$$

Obviously, any binary boolean function $\chi \not \equiv \perp$ is representable as $\chi_{\Upsilon}$ for appropriate $\Upsilon$. For the following, observe that if we denote by $\|\chi\|$ the cardinality of $\{\vec{\sigma} \mid \chi(\vec{\sigma})=\top\}$ then $\left\|\chi_{\Upsilon}\right\|=|\Upsilon|$. Lemma 2.4 shows that prime logics are exhausted by $\boldsymbol{\Lambda}_{\Upsilon}$. In the sequel, we write $\boldsymbol{\Lambda}_{\perp \top}$ instead of $\boldsymbol{\Lambda}_{\{\perp, T\}}$ and similarly for other $\boldsymbol{\Lambda}_{\Upsilon}$ and $\chi_{\Upsilon}$.

Lemma 2.4 For any binary boolean function $\chi \not \equiv \perp$, there exists a logic $L$ such that the c.f. of $\square$ over $L$ equals $\chi$. In fact, the c.f. of $\square$ over $\boldsymbol{\Lambda}_{\Upsilon}$ is $\chi_{\Upsilon}$.

Proof. For every $\nabla \in \Upsilon, \boldsymbol{\Lambda}_{\nabla} \vdash \chi_{\Upsilon}(\square \perp, \square \top)$, for $\boldsymbol{\Lambda}_{\nabla} \vdash \square p \leftrightarrow \nabla p$ and so the $\nabla$-th disjunct of $\chi_{\Upsilon}(\square \perp, \square \top)$, i.e., $(\square \perp)^{\nabla \perp} \wedge(\square \top)^{\nabla \top}$ is equivalent to $T$ in $\boldsymbol{\Lambda}_{\nabla}$.

Further, if not $\chi_{\Upsilon} \leqslant f$ then, for some $\nabla \in \Upsilon$, a FDNF of $f$ does not contain the $\nabla$-th disjunct. But other disjuncts of $f(\square \perp, \square \top)$ are obviously equivalent to $\perp$ in $\boldsymbol{\Lambda}_{\nabla}$. Thus $f(\square \perp, \square \top)$ does not belong to $\boldsymbol{\Lambda}_{\nabla}$ and so to $\boldsymbol{\Lambda}_{\Upsilon}$. 


$$
\begin{aligned}
& \boldsymbol{\Lambda}_{\perp}=\mathbf{E}\{\square p \leftrightarrow \perp\} ; \quad \boldsymbol{\Lambda}_{\bigcirc}=\mathbf{E}\{\square p \leftrightarrow p\} ; \\
& \boldsymbol{\Lambda}_{\top}=\mathbf{E}\{\square p \leftrightarrow \top\} ; \quad \boldsymbol{\Lambda}_{\neg}=\mathbf{E}\{\square p \leftrightarrow \neg p\} . \\
& \boldsymbol{\Lambda}_{\perp \bigcirc}=\mathbf{E}\{\square p \leftrightarrow(p \wedge \square T)\} ; \quad \boldsymbol{\Lambda}_{\perp \neg}=\mathbf{E}\{\square p \leftrightarrow(\neg p \wedge \square \perp)\} ; \\
& \boldsymbol{\Lambda}_{\bigcirc \top}=\mathbf{E}\{\square p \leftrightarrow(p \vee \square \perp)\} ; \quad \boldsymbol{\Lambda}_{\neg \top}=\mathbf{E}\{\square p \leftrightarrow(\neg p \vee \square T)\} ; \\
& \boldsymbol{\Lambda}_{\bigcirc\urcorner}=\mathbf{E}\{\square p \leftrightarrow(p \leftrightarrow \square \top)\} ; \quad \boldsymbol{\Lambda}_{\perp \top}=\mathbf{E}\{\square p \leftrightarrow \square \perp\} . \\
& \boldsymbol{\Lambda}_{\perp \bigcirc \neg}=\mathbf{E}\{\square p \leftrightarrow[(p \leftrightarrow \square \top) \wedge(\neg p \leftrightarrow \square \perp)]\} ; \\
& \boldsymbol{\Lambda}_{\bigcirc \neg \top}=\mathbf{E}\{\square p \leftrightarrow[(p \leftrightarrow \square T) \vee(\neg p \leftrightarrow \square \perp)]\} ; \\
& \boldsymbol{\Lambda}_{\perp \bigcirc \top}=\mathbf{E}\{\square p \leftrightarrow[\square \perp \vee(\square T \wedge p)]\} ; \\
& \Lambda_{\perp \neg T}=\mathbf{E}\{\square p \leftrightarrow[\square \top \vee(\square \perp \wedge \neg p)]\} . \\
& \boldsymbol{\Lambda}_{\perp \bigcirc \neg \top}=\mathbf{E}\{\square p \leftrightarrow[(p \wedge \square \top) \vee(\neg p \wedge \square \perp)]\} .
\end{aligned}
$$

Figure 1: Axiomatisation of the prime logics.

Theorem 2.5 The prime logics have the axiomatisation shown in Figure 1.

Proof. The inclusions $(\supseteq)$ are easily verified by the definition of $\boldsymbol{\Lambda}_{\Upsilon}$.

$(\subseteq)|\Upsilon|=1$. Fix $\nabla \in\{\perp, \bigcirc, \neg, \top\}$ and denote $\mathbf{E}_{\nabla}:=\mathbf{E}\{\square p \leftrightarrow \nabla p\}$. By induction on $A, \mathbf{E}_{\nabla} \vdash A \leftrightarrow \operatorname{tr}_{\nabla}(A)$. So, if $A \in \boldsymbol{\Lambda}_{\nabla}$ then $\boldsymbol{\Lambda}_{\nabla} \vdash \operatorname{tr}_{\nabla}(A)$ but $\operatorname{tr}_{\nabla}(A)$ has no $\square$ s, hence it is a tautology and thus belongs to $\mathbf{E}_{\nabla}$, whence $A \in \mathbf{E}_{\nabla}$.

$|\Upsilon|>1$. We use the following: if formulas $A$ and $B$ have no variables in common then $\mathbf{E}\{A\} \cap \mathbf{E}\{B\}=\mathbf{E}\{A \vee B\}$. Now to prove the needed inclusion for, say

$$
\boldsymbol{\Lambda}_{\perp \bigcirc}=\mathbf{E}_{\perp} \cap \mathbf{E}_{\bigcirc}=\mathbf{E}\{(\square p \leftrightarrow \perp) \vee(\square q \leftrightarrow q)\},
$$

note that replacing all subformulas of the form $\square A$ in $(\square p \leftrightarrow \perp) \vee(\square q \leftrightarrow q)$ by $A \wedge \square \top$ (i.e., the r.h.s. of the presumed axiom of $\boldsymbol{\Lambda}_{\perp \mathrm{O}}$ ) yields a tautology.

\section{Measuring expressibility of logics}

By $\varepsilon(L)$ (resp., $\alpha(L)$ ) we denote the number of modalities which are pairwise nonequivalent in (resp., non-analogous over) a logic $L$ (in the sequel, we usually omit the word 'pairwise' in these contexts). These are either natural numbers or the symbol $\infty$ and may be regarded as measures of expressiveness for $L$.

In this section we calculate $\varepsilon(L)$ and $\alpha(L)$ for the prime logics, some normal logics, and the provability logics. We also establish the iterativity of the logic $\mathbf{B}$.

We begin with some general observations. For any logic $L$, we have $\varepsilon(L) \geqslant 4$ and $\alpha(L) \geqslant 4$, since the logics of $\perp, \bigcirc, \neg$, and $T$ are distinct over $L$. If $L$ is closed under (RE) then also $\alpha(L) \leqslant \varepsilon(L)$. We shall see that $\alpha(L)<\varepsilon(L)$ for some $L$. However, we have no example of a logic $L$ with $\varepsilon(L)=\infty$ and $\alpha(L)<\infty$. Moreover, $\varepsilon(\cdot)$ is antimonotone, i.e., if $M \subseteq L$ then $\varepsilon(M) \geqslant \varepsilon(L)$. So far we do not know whether the same holds for $\alpha(\cdot)$, even on the class of logics that are closed under (RE). The following lemma is a step towards the answer to this question. 
Lemma 3.1 If $M$ is a logic closed under (RE) and $M \subseteq L$ then $\varepsilon(M) \geqslant \alpha(L)$.

Proof. It suffices to prove that if two modalities are equivalent in $M$ then they are analogous over $L$. Assume that $M \vdash \nabla p \leftrightarrow \Delta p$. Then by induction on a formula $A$, one can show that $M \vdash \operatorname{tr}_{\nabla}(A) \leftrightarrow \operatorname{tr}_{\Delta}(A)$. Hence $L \vdash \operatorname{tr}_{\nabla}(A)$ iff $L \vdash \operatorname{tr}_{\Delta}(A)$. Thus $L(\nabla)=L(\Delta)$.

Furthermore, $\varepsilon(L)$ is the cardinality of a boolean algebra, so by Stone's theorem, it is either $\infty$ or $2^{n}$ for some $n>0$. On the contrary, we shall see that $\alpha(L)$ may be an odd number. An example is $\alpha\left(\boldsymbol{\Lambda}_{\perp \bigcirc \neg \top}\right)=15$.

\subsection{Expressibility of prime logics}

Lemma 3.2 Suppose $\nabla$ is a proper constant in a logic $L$ and $\Delta$ is a boolean combination of $\bigcirc$ and $\nabla$. Then:

(1) $L(\Delta)=\boldsymbol{\Lambda}_{\Upsilon}$ for some $\Upsilon$ with $|\Upsilon| \leqslant 2$;

(2) moreover, if $\Delta$ is "read off" from the r.h.s. of the axiom of $\boldsymbol{\Lambda}_{\Upsilon}$ for some $\Upsilon$ with $|\Upsilon|=2$ (e.g., for $\boldsymbol{\Lambda}_{\perp \neg}$ take $\left.\Delta=\neg \bigcirc \wedge \nabla\right)$ then $L(\Delta)=\boldsymbol{\Lambda}_{\Upsilon}$;

(3) in particular, $L(\nabla)=\boldsymbol{\Lambda}_{\perp \top}$ (the logic of a proper constant is the intersection of the logics of two trivial constants $\perp$ and $\top$ ).

Proof. (1) Any such $\Delta$ is equivalent (and hence analogous, since $\Delta$ is prime) to a modality mentioned in item (2).

(2) To prove that the logic of a modality, say, $\Delta:=\bigcirc \wedge \nabla$ over $L$ is $\boldsymbol{\Lambda}_{\perp \bigcirc}$ observe that the c.f. of $\Delta$ over $L$ is $\chi(x, y) \equiv x \equiv \chi_{\perp \bigcirc}(x, y)$.

Definition 3.3 Constants $\nabla$ and $\Delta$ are independent in a logic $L$ if, for any binary boolean function $f(x, y), L \vdash f(\nabla, \Delta)$ implies $f(x, y) \equiv \top$; in other words, if the c.f. of a modality induced by $(\neg p \wedge \nabla) \vee(p \wedge \Delta)$ over $L$ equals $T$.

Lemma 3.4 $\Lambda_{\Upsilon} \hookrightarrow \Lambda_{\Upsilon^{\prime}}$ iff $|\Upsilon| \leqslant\left|\Upsilon^{\prime}\right|$, for any $\varnothing \neq \Upsilon, \Upsilon^{\prime} \subseteq\{\perp, \bigcirc, \neg, \top\}$.

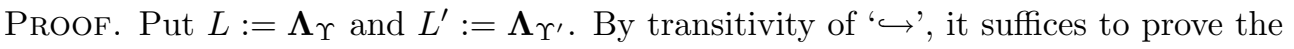
following claims:

(a) $L \hookrightarrow L^{\prime}$ for every $\Upsilon, \Upsilon^{\prime}$ with $|\Upsilon|=\left|\Upsilon^{\prime}\right|$;

(b) $L \hookrightarrow L^{\prime}$ for some $\Upsilon, \Upsilon^{\prime}$ with $|\Upsilon|=\left|\Upsilon^{\prime}\right|-1$;

(c) $L \nrightarrow L^{\prime}$ for some $\Upsilon, \Upsilon^{\prime}$ with $|\Upsilon|=\left|\Upsilon^{\prime}\right|+1$.

The cases $|\Upsilon|=1$ in (a), (b), $|\Upsilon|=4$ in (a), and $\left|\Upsilon^{\prime}\right|=1$ in (c) are trivial.

(a) $|\Upsilon|=2$. A logic $L^{\prime}$ has a proper constant $\nabla$ (namely, the one occurring in the r.h.s. of the axiom of $L^{\prime}$ ). Hence the claim follows from Lemma 3.2(2).

$|\Upsilon|=3$. To prove that, say $L:=\boldsymbol{\Lambda}_{\perp \bigcirc \top} \hookrightarrow \boldsymbol{\Lambda}_{\bigcirc \neg \top}=: L^{\prime}$, note that by Lemma 2.4, the c.f. of $\square$ over $L^{\prime}$ is $x \vee y$, i.e., $L^{\prime} \vdash \square \perp \vee \square T$. Then the c.f. of a modality $\nabla$ induced by $(\neg p \wedge \neg \square \perp) \vee(p \wedge \square \top)$ is $\neg x \vee y$, since $L^{\prime} \vdash \nabla \perp \leftrightarrow \neg \square \perp$ and $L^{\prime} \vdash \nabla \top \leftrightarrow \square \top$. Therefore, $L^{\prime}(\nabla)=L$.

(b) $|\Upsilon|=2$. The constant $\square \top$ is proper in $L^{\prime}:=\boldsymbol{\Lambda}_{\perp \bigcirc_{\neg}}$, so $L^{\prime}(\square \top)=\boldsymbol{\Lambda}_{\perp \top}$.

$|\Upsilon|=3$. By Theorem 2.5, $L=\mathbf{E}\{\square p \leftrightarrow \nabla p\}$ for a prime modality $\nabla$. Then the c.f. of $\square$ over $L$ equals the c.f. of $\nabla$ over $L^{\prime}:=\Lambda_{\perp \bigcirc \neg \top}$, so $L^{\prime}(\nabla)=L$. 
(c) $|\Upsilon|=3$. By Theorem 2.5, any modality $\nabla$ is equivalent in $L^{\prime}:=\boldsymbol{\Lambda}_{\perp \top}$ to a boolean combination of $\bigcirc$ and the proper constant $\square \perp$, hence by Lemma 3.2(1), $L^{\prime}(\nabla)=\Lambda_{\Upsilon}$ for some $\Upsilon$ with $|\Upsilon| \leqslant 2$.

$|\Upsilon|=4$. It is easily seen that $L^{\prime}:=\boldsymbol{\Lambda}_{\perp \bigcirc \top}$ has no independent constants, hence no modalities with a c.f. $\top$. Therefore, $\boldsymbol{\Lambda}_{\perp \bigcirc \neg \top} \nrightarrow L^{\prime}$.

Theorem 3.5 For $|\Upsilon|=1$, 2, 3, 4, we have $\varepsilon\left(\boldsymbol{\Lambda}_{\Upsilon}\right)=4$, 16, 64, 256, whereas $\alpha\left(\boldsymbol{\Lambda}_{\Upsilon}\right)=4,10,14,15$.

Proof. The claim for $\alpha$ follows from Lemma 3.4 and the fact that every modality is equivalent in (and hence analogous over) $\boldsymbol{\Lambda}_{\Upsilon}$ to a prime modality.

Any modality is equivalent in $L:=\boldsymbol{\Lambda}_{\Upsilon}$ to a boolean combination of $\bigcirc, \square \perp$, and $\square$ T; there are exactly 256 such combinations. Take any two of them, $b_{1}$ and $b_{2}$, and put $b:=\left(b_{1} \leftrightarrow b_{2}\right)$. Clearly, $L \vdash b_{1} \leftrightarrow b_{2}$ iff $L \vdash b(p, \square \perp, \square \top)$, iff $L \vdash b(\sigma, \square \perp, \square \top)$, for all $\sigma \in \mathbf{2}$, iff $b(\sigma, x, y) \geqslant \chi_{\Upsilon}(x, y)$, for all $\sigma \in \mathbf{2}$, iff functions $b_{1}(t, x, y)$ and $b_{2}(t, x, y)$ have equal restrictions to the set $\left\{\left(\sigma, \delta_{0}, \delta_{1}\right) \in \mathbf{2}^{3} \mid \chi_{\Upsilon}\left(\delta_{0}, \delta_{1}\right)=\top\right\}$ of cardinality $2\left\|\chi_{\Upsilon}\right\|=2|\Upsilon|$. Thus $\varepsilon(L)=2^{2|\Upsilon|}$.

\subsection{Expressibility of normal logics}

Definition 3.6 A normal logic (cf. [3,4]) is a set of formulas containing the axioms $(A \top)$ and $(A K)$ shown in Figure 2 and closed under (MP), (Sub), and the rule of necessitation:

$$
\text { (Nec) } \frac{A}{\square A}
$$

Clearly, every normal logic is closed under the rule (RE). Moreover, a logic containing $(A K)$ and $\square \top$ is normal iff it is closed under (RE).

$$
\begin{array}{ll}
(A \top) \text { Tautologies in the modal language } \\
(A K) \square(p \rightarrow q) \rightarrow(\square p \rightarrow \square q) & \text { (distributivity) } \\
(A T) \square p \rightarrow p & \text { (reflexivity) } \\
(A 4) \square p \rightarrow \square \square p & \text { (transitivity) } \\
(A B) p \rightarrow \square \diamond p & \text { (symmetry) } \\
(A 5) \diamond p \rightarrow \square \diamond p & \text { (euclideanness) } \\
(A L) \square(\square p \rightarrow p) \rightarrow \square p & \text { (Löb axiom) } \\
(A G) \square(\square(p \rightarrow \square p) \rightarrow p) \rightarrow p & \text { (Grzegorczyk axiom) }
\end{array}
$$

Figure 2: Axioms of normal logics

We shall mainly concerned with the well-known normal logics which are axiomatised as follows (extra axioms are shown in Figure 2).

$$
\begin{array}{rlrl}
\mathbf{K} & =(A \top)+(A K)+(\mathrm{MP})+(\mathrm{Sub})+(\mathrm{Nec}), \\
\mathbf{T} & =\mathbf{K}+(A T), & \mathbf{B} & =\mathbf{T}+(A B), \\
\mathbf{K} 4 & =\mathbf{K}+(A 4), & \mathbf{S 4} & =\mathbf{T}+(A 4), \\
\mathbf{K 5} & =\mathbf{K}+(A 5), & \mathbf{S 5} & =\mathbf{T}+(A 5), \\
\mathbf{K B} & =\mathbf{K}+(A B), & \mathbf{K 4 5} & =\mathbf{K} 4+(A 5), \\
\mathbf{G L} & =\mathbf{K}+(A L), & \mathbf{G r z} & =\mathbf{K}+(A G) .
\end{array}
$$


We shall consider also the so called Diodorean logic $\mathbf{D}^{*}$ (cf. [11]), which is the set of all formulas that are valid on the frame $(\omega, \geqslant)$, or equivalently, the logic of the class of finite reflexive transitive linear orders.

The following strict inclusions hold between these logics:

$$
\begin{aligned}
& \mathbf{K} \subset \mathbf{K 5} \quad \mathbf{K} \subset \mathbf{K} \mathbf{4} \subset \mathbf{G L}
\end{aligned}
$$

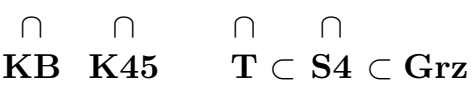

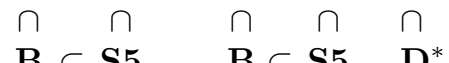

In what follows, we use Kripke semantics of normal logics. All necessary definitions and facts can be found in $[3,4]$, and we use them without explicit references. Usually we denote a frame by $(W, \uparrow)$, where $\uparrow$ stands for an accessibility relation on a set of worlds $W$. A set of worlds accessible from $w \in W$ is denoted by $w \uparrow:=\{x \in W \mid w \uparrow x\}$; the symbol $\downarrow$ stands for the inverse relation of $\uparrow$. Recall that a logic $L$ is called (Kripke) complete (w.r.t. a class of frames $\mathcal{F}$ ) if $A \in L$ iff $\forall F \in \mathcal{F} F \models A$, for all $A \in \mathrm{Fm}$.

Definition 3.7 A sequence of modalities $\nabla_{n}, n \geqslant 1$, is strong (hereditary strong) if, for any complete logic $L$, if $\nabla_{n}$ are non-equivalent in $L$ then they are non-analogous over $L$ (over any logic $M \in[\mathbf{K}, L]$ ).

Let $[1, \infty):=\{1,2, \ldots\}$. For any $X \subseteq[1, \infty)$ put

$$
X_{i}:= \begin{cases}\top, & \text { if } i \in X \\ \perp, & \text { if } i \notin X\end{cases}
$$

and denote by $X \downarrow:=\{n-1 \mid 1<n \in X\}$ the left shift of $X$ (with $X_{1}$ lost). Recall that $p^{\perp}=\neg p$ and $p^{\top}=p$. We define sequences of modalities $\nabla_{n}^{X}$ simultaneously for all $X \subseteq[1, \infty)$ by induction on $n$ :

$$
\nabla_{1}^{X} p:=p^{X_{1}} ; \quad \nabla_{n+1}^{X} p:=p^{X_{1}} \wedge \diamond \nabla_{n}^{X \downarrow} p .
$$

Remark 3.8 Obviously, for any $X \subseteq[1, \infty), \mathbf{K} \vdash \nabla_{1}^{X} p \leftarrow \nabla_{2}^{X} p \leftarrow \nabla_{3}^{X} p \leftarrow \ldots$

Take $N \geqslant m \geqslant 1, \mathcal{N}:=\{1, \ldots, N\}$, distinct variables $p_{1}, \ldots, p_{N}$ and define

$$
A_{N}^{m}:=\square\left(\bigvee_{j \in \mathcal{N}} p_{j}\right) \longrightarrow \bigvee_{\substack{\mathcal{J} \subseteq \mathcal{N} \\|\mathcal{J}|=m}} \square\left(\bigvee_{j \in \mathcal{J}} p_{j}\right)
$$

Theorem 3.9 For $X=[1, \infty)$, the sequence $\nabla_{n}:=\nabla_{n}^{X}$ is hereditary strong.

Proof. Suppose $\nabla_{n}$ are non-equivalent in a complete logic $L$.

Lemma 3.10 If $m \geqslant n$ then $\mathbf{K}\left(\nabla_{n}\right) \vdash A_{N}^{m}$, for all $N \geqslant m$.

- Recall that $\mathbf{K}$ is complete w.r.t. the class of all frames. Take any model $(W, \uparrow, \models)$ and $x_{1} \in W$. To prove that $x_{1} \models \operatorname{tr}_{\nabla_{n}}\left(A_{N}^{m}\right)$, assume that $x_{1} \models \nabla_{n} \bigvee_{j \in \mathcal{N}} p_{j}$. Then there is a chain $x_{1} \uparrow x_{2} \uparrow \ldots \uparrow x_{n}$ such that $\forall i, 1 \leqslant i \leqslant n, \exists j=j(i) \in \mathcal{N} x_{i} \models p_{j}$. Taking any $\mathcal{J} \subseteq \mathcal{N}$ such that $\mathcal{J} \supseteq\{j(1), \ldots, j(n)\}$ and $|\mathcal{J}|=m$, we obtain $x_{1} \models \nabla_{n} \bigvee_{j \in \mathcal{J}} p_{j}$ 
Lemma 3.11 If $m<n$ then $L\left(\nabla_{n}\right) \nvdash A_{N}^{m}$, for any $N \geqslant n$.

- Since $L \vdash \nabla_{n+1} p \rightarrow \nabla_{n} p$ by Remark 3.8, we have $L \forall \nabla_{n} p \rightarrow \nabla_{n+1} p$. By completeness of $L$, there exists an $L$-frame $F=(W, \uparrow)$ and a valuation $\models$ (of $p$ only) such that $x_{1} \not \neq \nabla_{n} p \rightarrow \nabla_{n+1} p$ for some $x_{1} \in W$. To prove Lemma it suffices to find a valuation $\models^{\prime}$ of $p_{1}, \ldots, p_{N}$ such that $x_{1} \mid \neq \operatorname{tr}_{\nabla_{n}}\left(A_{N}^{m}\right)$, since by virtue of $F \models L$, this will imply $L\left(\nabla_{n}\right) \nvdash A_{N}^{m}$.

As $x_{1} \models \nabla_{n} p$, there is a chain $x_{1} \uparrow x_{2} \uparrow \ldots \uparrow x_{n}$ such that $\forall i, 1 \leqslant i \leqslant n, x_{i} \models p$. Now define $\Perp^{\prime}$ by putting $x_{i} \Perp^{\prime} p_{i}$, for every $i, 1 \leqslant i \leqslant n$.

Clearly, $x_{1} \stackrel{\models}{=} \nabla_{n} \bigvee_{j \in \mathcal{N}} p_{j}$, since each $x_{i}$ validates at least one of the $p_{j}$. However, for any $\mathcal{J} \subset \mathcal{N}$ with $|\mathcal{J}|=m$, we have $x_{1} \mid \neq \nabla_{n} \bigvee_{j \in \mathcal{J}} p_{j}$. For assume the converse, then there is a chain $x_{1}=x_{1}^{\prime} \uparrow x_{2}^{\prime} \uparrow \ldots \uparrow x_{n}^{\prime}$ such that $\forall i, 1 \leqslant i \leqslant n, \quad \exists j=j(i) \in \mathcal{J}$ $x_{i}^{\prime} \models^{\prime} p_{j(i)}$. By definition of $\models^{\prime}$, this implies $\left\{x_{1}^{\prime}, \ldots, x_{n}^{\prime}\right\} \subseteq\left\{x_{1}, \ldots, x_{n}\right\}$. As $x_{1} \not \models$ $\nabla_{n+1} p$, there is no chain beginning at $x_{1}$ and consisting of $n+1$ worlds satisfying $p$. In particular, for any $1 \leqslant i \leqslant j \leqslant n$, we have $\neg\left(x_{j} \uparrow x_{i}\right)$. Hence, there exists a unique chain consisting of $n$ worlds belonging to the set $\left\{x_{1}, \ldots, x_{n}\right\}$, namely, $x_{1} \uparrow \ldots \uparrow x_{n}$. Thus $x_{i}^{\prime}=x_{i}$ and $j(i)=i$, for all $i, 1 \leqslant i \leqslant n$, so $\{j(1), \ldots, j(n)\}=\{1, \ldots, n\} \subseteq \mathcal{J}$, which contradicts to $|\mathcal{J}|=m<n$.

The lemmas imply, for any logic $M \in[\mathbf{K}, L]$, that if $N \geqslant \max (m, n)$ then $M\left(\nabla_{n}\right) \vdash$ $A_{N}^{m}$ iff $m \geqslant n$. Therefore, the logics $M\left(\nabla_{n}\right)$ are distinct.

Corollary $3.12 \varepsilon(L)=\alpha(L)=\infty$, for any logic $L \in[\mathbf{K}, \mathbf{G L}]$.

Proof. GL is complete w.r.t. finite irreflexive transitive trees. So, to see that modalities $\nabla_{n}$ from Theorem 3.9 are non-equivalent in $\mathbf{G L}$, take a frame $F=(\{1, \ldots, n\},<)$ and put $i=p$, for all $i, 1 \leqslant i \leqslant n$. Then $F \models \mathbf{G L}$ but $1 \not \neq \nabla_{n} p \rightarrow \nabla_{n+1} p$. Thus, firstly, $\varepsilon(\mathbf{G L})=\infty$ and hence $\varepsilon(L)=\infty$; secondly, by Theorem $3.9, \alpha(L)=\infty$, for any $L \in[\mathbf{K}, \mathbf{G L}]$.

In comparison with this result, consider linear modalities, i.e., sequences of $\square \mathrm{s}$ and $\neg \mathrm{s}$ (not containing the subsequence $\neg \neg$, without loss of generality).

Theorem 3.13 There are exactly 7 linear modalities which are non-analogous over GL, namely, $\bigcirc, \neg, \square, \square \neg, \neg \square, \diamond$, and $\square \neg \square$.

Proof. First observe that, for any linear modality $\nabla$ containing a subsequence $\square \neg \square$, $\mathbf{G L}(\nabla)=\boldsymbol{\Lambda}_{\perp T}$. Indeed, $\nabla$ has a form $\square^{m} \diamond \Delta$ or $\neg \square^{m} \diamond \Delta$ for some $\Delta$ and $m \geqslant 1$. But GL $\vdash \square^{m} \diamond A \leftrightarrow \square^{m} \perp$, so $\nabla$ is equivalent in GL to a proper constant $\square^{m} \perp$ or $\neg \square^{m} \perp$, hence $\mathbf{G L}(\nabla)=\boldsymbol{\Lambda}_{\perp \top}$, by Lemma 3.2(3).

Next recall that $\mathbf{G L}$ is iterative (cf. [1]), i.e., $\mathbf{G L}\left(\square^{n}\right)=\mathbf{G L}$, for all $n>0$. Finally, it is easily seen that if modalities $\nabla$ and $\Delta$ are analogous over any logic $L$ then so are $\neg \nabla$ and $\neg \Delta$, as well as $\nabla \neg$ and $\Delta \neg$, as well as $\neg \nabla \neg$ and $\neg \Delta \neg$.

From these facts the theorem follows immediately.

If a logic $L$ contains the reflexivity axiom $(A T)$ then the modalities from Theorem 3.9 are equivalent in $L$ to each other, so the theorem cannot be applied to establish $\alpha(L)=\infty$. The remedy is to generalise the theorem.

First, we generalise Lemma 3.10. Suppose $|X|=\infty$ and choose numbers $0=n_{0}<$ $n_{1}<n_{2}<\ldots$ so that $X \cap\left(n_{k}, n_{k+1}\right] \neq \varnothing$, for all $k \geqslant 0$, where we use a notation 
$(r, t]:=\{s \in \omega \mid r<s \leqslant t\}$. Consider the sequence $\nabla_{k}:=\nabla_{n_{k}}^{X}, k \geqslant 1$. Observe that $\left|X \cap\left(n_{k}, n_{k+1}\right]\right|$ is the number of positive occurrences of $p$ in $\nabla_{k} p$, thus the condition $X \cap\left(n_{k}, n_{k+1}\right] \neq \varnothing$ merely means that the number of positive occurrences of $p$ in $\nabla_{k} p$ increases as $k$ increases.

Lemma 3.14 If $m \geqslant\left|X \cap\left(0, n_{k}\right]\right|$ then $\mathbf{K}\left(\nabla_{k}\right) \vdash A_{N}^{m}$, for all $N \geqslant m$.

Proof. Take any model $(W, \uparrow, \mid=)$ and $x_{1} \in W$. To prove that $x_{1} \models \operatorname{tr}_{\nabla_{k}}\left(A_{N}^{m}\right)$, assume that $x_{1}=\nabla_{k} \bigvee_{j \in \mathcal{N}} p_{j}$. Then there is a chain $x_{1} \uparrow x_{2} \uparrow \ldots \uparrow x_{n_{k}}$ such that $\forall i$, $1 \leqslant i \leqslant n_{k}$, we have: if $i \in X$ then $x_{i} \models p_{j}$ for some $\exists j=j(i) \in \mathcal{N}$, else $x_{i} \not \models p_{j}$, for any $j \in \mathcal{N}$. Now take any $\mathcal{J} \subseteq \mathcal{N}$ with $|\mathcal{J}|=m$ (it is possible, since $N \geqslant m$ ) such that $\mathcal{J} \supseteq\left\{j(i) \mid i \in X \cap\left(0, n_{k}\right]\right\}$ and obtain $x_{1} \models \nabla_{n} \bigvee_{j \in \mathcal{J}} p_{j}$

We have succeeded in generalising Lemma 3.11 only to the case when $X$ is an infinite arithmetical progression

$$
X=\{a+d i \mid i \geqslant 0\}, \text { where } d \geqslant 1, a \geqslant 1 .
$$

Suppose $\nabla_{k}$ are non-equivalent in a complete logic $L$.

Lemma 3.15 If $m<n:=\left|X \cap\left(0, n_{k}\right]\right|$ then $L\left(\nabla_{k}\right) \forall A_{N}^{m}$, for any $N \geqslant n$.

Proof. As above, for some $L$-frame $F=(W, \uparrow)$, a valuation $\models$ of $p$, and $x_{1} \in W$, we have $x_{1} \not \neq \nabla_{k} p \rightarrow \nabla_{k+1} p$. Again, it suffices to find a valuation $\left.\right|^{\prime}$ of $p_{1}, \ldots, p_{N}$ such that $x_{1} \|^{\prime} \operatorname{tr}_{\nabla_{k}}\left(A_{N}^{m}\right)$.

Since $x_{1} \models \nabla_{k} p$, there is a chain $x_{1} \uparrow \ldots \uparrow x_{n_{k}}$ such that $x_{i} \models p$ iff $i \in X$, for all $i$, $1 \leqslant i \leqslant n_{k}$, i.e., among $x_{i}$ there are exactly $n$ worlds validating $p$; denote them by $y_{\ell}:=x_{i_{\ell}}, 1 \leqslant \ell \leqslant n$, where $i_{1}<\ldots<i_{n},\left\{i_{1}, \ldots, i_{n}\right\}=X \cap\left[1, n_{k}\right]$, and $y_{\ell} \models p$.

Now define $\left.\right|^{\prime}$ by putting $y_{\ell} \models^{\prime} p_{\ell}$, for every $\ell, 1 \leqslant \ell \leqslant n$. Clearly, $x_{1} \stackrel{\prime}{=} \nabla_{k} \bigvee_{j \in \mathcal{N}} p_{j}$, since each $y_{\ell}$ validates at least one of $p_{j}$. But, for any $\mathcal{J} \subseteq \mathcal{N}$ with $|\mathcal{J}|=m$, we claim that $x_{1} \not^{\prime} \nabla_{n} \bigvee_{j \in \mathcal{J}} p_{j}$. Otherwise, there is a chain $x_{1}=x_{1}^{\prime} \uparrow x_{2}^{\prime} \uparrow \ldots \uparrow x_{n_{k}}^{\prime}$ such that $\forall \ell, 1 \leqslant \ell \leqslant n, \exists j=j(\ell) \in \mathcal{J} \quad x_{i_{\ell}}^{\prime} \models^{\prime}=p_{j(\ell)}$. By definition of $\models^{\prime},\left\{x_{i_{1}}^{\prime}, \ldots, x_{i_{n}}^{\prime}\right\} \subseteq$ $\left\{y_{1}, \ldots, y_{n}\right\}$. Furthermore, $x_{i_{1}}^{\prime} \uparrow^{d} \ldots \uparrow^{d} x_{i_{n}}^{\prime}$ and $y_{1} \uparrow^{d} \ldots \uparrow^{d} y_{n}$. Arguing as in Lemma 3.11, we have $\neg\left(y_{j} \uparrow^{d} y_{i}\right)$, for all $1 \leqslant i \leqslant j \leqslant n$. Hence $x_{i_{\ell}}^{\prime}=y_{\ell}$ and $j(\ell)=\ell$, for all $\ell, 1 \leqslant \ell \leqslant n$, thus $\{j(1), \ldots, j(n)\}=\{1, \ldots, n\} \subseteq \mathcal{J}$ in contradiction with $|\mathcal{J}|=m<n$.

Theorem 3.16 Suppose $X \subseteq[1, \infty)$ is an infinite arithmetical progression and numbers $0=n_{0}<n_{1}<n_{2}<\ldots$ satisfy $X \cap\left(n_{k}, n_{k+1}\right] \neq \varnothing$, for all $k \geqslant 0$. Then a sequence $\nabla_{k}:=\nabla_{n_{k}}^{X}, k \geqslant 1$, is hereditary strong.

Proof. Similar to the proof of Theorem 3.9, using Lemmas 3.14 and 3.15.

Corollary $3.17 \varepsilon(L)=\alpha(L)=\infty$, for any logic $L \in\left[\mathbf{K}, \mathbf{D}^{*}\right]$.

Proof. Let $X$ be the set of odd natural numbers and $n_{k}:=2 k$. Then $\mid X \cap$ $\left(n_{k}, n_{k+1}\right] \mid=1$ and modalities $\nabla_{k}:=\nabla_{n_{k}}^{X}$ are non-equivalent in $\mathbf{D}^{*}$. To see the latter, put $F=(\{1, \ldots, 2 k\}, \leqslant)$ and $i=p$ iff $i$ is odd, for all $i, 1 \leqslant i \leqslant 2 k$. Then $F \models \mathbf{D}^{*}$ but $1 \not \neq \nabla_{k} p \rightarrow \nabla_{k+1} p$. 
Now we pass to the logic $\mathbf{K B}$, which it complete w.r.t. symmetric frames. For each infinite arithmetical progression $X \subseteq[1, \infty)$, a sequence $\nabla_{n}^{X}$ collapses in $\mathbf{K B}$ into a finite number of cosets modulo equivalence in $\mathbf{K B}$.

Now put $X=\{1,2,5,6,9,10, \ldots\}=\{4 i+1,4 i+2 \mid i \geqslant 0\}, n_{k}:=4 k$, and consider a sequence $\nabla_{k}:=\nabla_{n_{k}}^{X}, k \geqslant 1$.

To see that $\nabla_{k}$ are non-equivalent in $\mathbf{K B}$, put $F=(\{1, \ldots, 4 k\}, \uparrow)$, where $i \uparrow j$ iff $|i-j|=1$, and $i \models p$ iff $i \in X$. Then $F \models \mathbf{K B}$ but $1 \not \neq \nabla_{n} p \rightarrow \nabla_{n+1} p$.

We do not know whether the sequence $\nabla_{k}$ is (hereditary) strong. Moreover, all examples of (hereditary) strong sequences we know are covered by the ones mentioned in Theorem 3.16. But situation is not hopeless, for instead of quantifying over all complete logics as is done in the definition of a (hereditary) strong sequence, we can confine to a particular complete logic, say $\mathbf{K B}$, and prove the hereditary strength of $\nabla_{k}$ "relative" to this logic. This way leads to success, as the following theorem demonstrates.

Theorem $3.18 \varepsilon(L)=\alpha(L)=\infty$, for any logic $L \in[\mathbf{K}, \mathbf{K B}]$.

Proof. We only need to prove Lemma 3.15 with $\mathbf{K B}$ in place of $L$. Put $n:=$ $\left|X \cap\left[1, n_{k}\right]\right|=2 k$.

Lemma 3.19 If $m<n$ then $\mathbf{K B}\left(\nabla_{k}\right) \nvdash A_{N}^{m}$, for any $N \geqslant n$.

- Unlike the proof of Lemma 3.15, now it suffices to find any symmetric frame falsifying $\operatorname{tr}_{\nabla_{k}}\left(A_{N}^{m}\right)$. For this the above-mentioned $\mathbf{K B}$-frame $F$ suits, if we put variables $p_{1}, \ldots, p_{n}$ to be true at successive worlds of a set $X \cap\left(0, n_{k}\right]$. To be more exact, if $i_{1}<\ldots<i_{n}$ and $\left\{i_{1}, \ldots, i_{n}\right\}=X \cap\left(0, n_{k}\right]$ then we put $i_{\ell} \models p_{\ell}$, for all $\ell, 1 \leqslant \ell \leqslant n$. It is easily seen that $1 \not \neq \operatorname{tr}_{\nabla_{k}}\left(A_{N}^{m}\right)$.

The theorem follows immediately from Lemma 3.14 and 3.19.

We postpone the consideration of the logic $\mathbf{B}$ till the next subsection. Now we come to logics of "finite expressibility".

Theorem $3.20 \varepsilon($ S5 $)=\alpha($ S5 $)=16$.

Proof. Any modality is equivalent in S5 to one of the following 16:

(1) $\nabla, \neg \nabla, \nabla \neg, \neg \nabla \neg$, where $\nabla$ is either $\square$ or $\boxplus:=\bigcirc \rightarrow \square$;

(2) $\nabla, \neg \nabla$, where $\nabla$ is either $\bigcirc$ or $\odot:=\boxplus \wedge \diamond$;

(3) $\nabla, \neg \nabla$, where $\nabla$ is either $\perp$ or $\Delta:=\square \vee \square \neg$.

Now we show that the logics of these modalities (over S5) are distinct. The logics of $\perp, \bigcirc, \neg$, and $\top$ are distinct and they differ from logics of other modalities. The logics of modalities in item (2) and of no others contain the formula $\square \neg p \leftrightarrow \neg \square p$. The same holds for item (3) and the formula $\square \neg p \leftrightarrow \square p$. Furthermore, $(\square p \leftrightarrow \square \square p) \in$ $\mathbf{S 5}(\neg \odot) \backslash S 5(\odot)$ and $\square \square p \in \mathbf{S 5}(\Delta) \backslash S 5(\neg \Delta)$, thus all modalities in items (2) and (3) are non-analogous over S5. It is even easier to verify the same for item (1).

Theorem $3.21 \varepsilon(L) \leqslant 2^{2^{17}}$ and $\alpha(L) \leqslant 2^{2^{17}}$, for any logic $L \supseteq \mathbf{K 5}$. 
Proof. By Lemma 3.1, it suffices to prove that $\varepsilon(\mathbf{K} 5) \leqslant 2^{2^{17}}$.

First we claim that if S5 $\vdash A \rightarrow B$ then K5 $\vdash \square A \rightarrow \square B$. Recall that K5 is complete w.r.t. Euclidean frames. For any Euclidean frame $(W, \uparrow)$ and $w \in W$, the restriction of $\uparrow$ to $w \uparrow$ is total, so the frame $(w \uparrow, \uparrow)$ validates $\mathbf{S} 5$ and hence the formula $A \rightarrow B$, whence $w \models \square(A \rightarrow B)$ and finally $w \models \square A \rightarrow \square B$, by the distributivity axiom $(A K)$.

Now, since in $\mathbf{S} \mathbf{5}$ every modality is equivalent to one of $\nabla_{1}, \ldots, \nabla_{16}$ mentioned in the proof of Theorem 3.20, it follows from the above that every modality is equivalent in $\mathbf{K 5}$ to a boolean combination of $\bigcirc, \square \nabla_{1}, \ldots, \square \nabla_{16}$. But there are no more than $2^{2^{17}}$ distinct combinations of this kind.

\subsection{On the logic B}

We shall prove two facts. On the one hand, Theorem 3.22 states that the sequence $\square^{n}$ collapses over $\mathbf{B}$ according to the external approach. On the other hand, Theorem 3.25 shows that $\mathbf{B}$ is "rich enough" from the viewpoint of the same approach.

Theorem 3.22 The logic $\mathbf{B}$ is iterative: $\mathbf{B}\left(\square^{n}\right)=\mathbf{B}$, for any $n \geqslant 2$.

Proof. ( $\supseteq$ ) The rules of $\mathbf{B}$ become admissible in $\mathbf{B}$ after the $\square^{n}$-translation. Here we show schematically that the axioms of $\mathbf{B}$ become derivable in $\mathbf{B}$ after the $\square^{n}$ translation.

$\begin{aligned}(A K) \mathbf{B} & \vdash \square^{n}(p \rightarrow q) \rightarrow \square^{n-1}(\square p \rightarrow \square q) \rightarrow \cdots \\ & \cdots \rightarrow \square\left(\square^{n-1} p \rightarrow \square^{n-1} q\right) \rightarrow\left(\square^{n} p \rightarrow \square^{n} q\right) .\end{aligned}$

$(A T) \mathbf{B} \vdash \square^{n} p \rightarrow \square^{n-1} p \rightarrow \cdots \rightarrow \square p \rightarrow p$.

$(A B) \mathbf{B} \vdash p \rightarrow \square \diamond p \rightarrow \square(\square \diamond) \diamond p \equiv \square^{2} \diamond^{2} p \rightarrow \cdots$ $\cdots \rightarrow \square^{n-1}(\square \diamond) \diamond^{n-1} p \equiv \square^{n} \diamond^{n} p$.

$(\subseteq)$ Assume that $\mathbf{B} \forall \neg A$. Then by the completeness of $\mathbf{B}$, there exists a reflexive symmetric model $(W, \Uparrow, \models)$ such that $w \models A$ for some $w \in W$. Recall that a $\Uparrow$-chain of length $k$ is a sequence of the form $x_{0} \Uparrow \ldots \Uparrow x_{k}$.

We endow $W$ with a metric induced by $\Uparrow$ by putting

$$
\varrho(x, y)=\min \left\{r \geqslant 0 \mid x \Uparrow^{r} y\right\} .
$$

Metric axioms are easily verified. Recall that a $\Uparrow$-ball of radius $r$ and center $x \in W$ is the set $x \Uparrow^{r}=\{y \in W \mid \varrho(x, y) \leqslant r\}$.

Denote $F^{n}:=\operatorname{tr}_{\square^{n}}(F)$, for any $F \in \mathrm{Fm}$. Now, to prove that $\mathbf{B} \not \forall \neg A^{n}$ we construct a model $(\bar{W}, \uparrow, \models)$ such that $A^{n}$ is true at some world of $\bar{W}$. The idea is to "disperse" each $x \in W$ to a $\uparrow$-ball of radius $m:=n-1$. Formally, we put

$$
\bar{W}=\left\{x, x_{1}^{y}, \ldots, x_{m}^{y} \mid x, y \in W, x \Uparrow y, x \neq y\right\} .
$$

Clearly, $W \subseteq \bar{W}$. Let $\uparrow$ be the least reflexive symmetric relation on $\bar{W}$ satisfying the following conditions (see Figure 3):

- for all distinct $x, y \in W$ there is a chain $x \uparrow x_{1}^{y} \uparrow \ldots \uparrow x_{m}^{y} \uparrow y_{m}^{x} \uparrow \ldots \uparrow y_{1}^{x} \uparrow y$; 


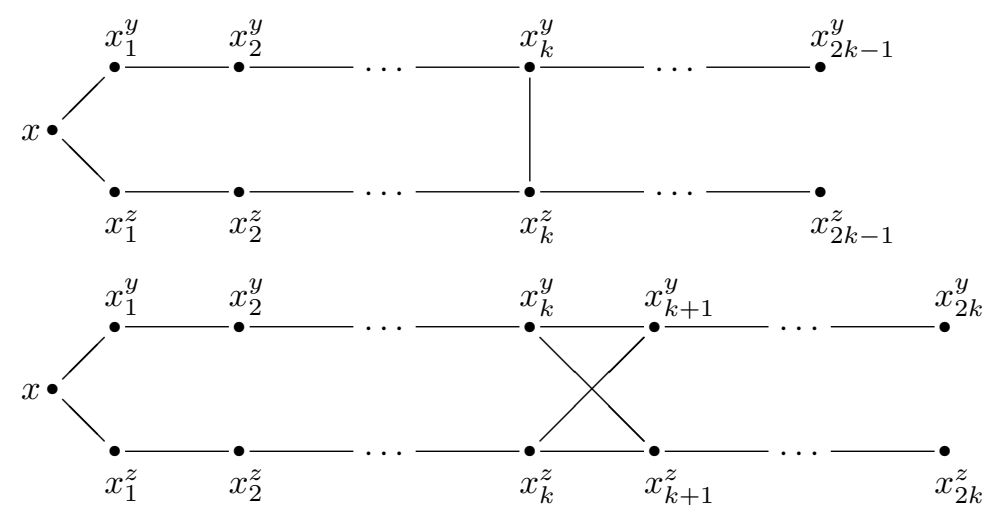

Figure 3: The relation $\uparrow$ for $n=2 k$ (upper) and $n=2 k+1$ (lower).

- for all $x \in W$ and distinct $y, z \in x \Uparrow, \begin{cases}x_{k}^{y} \uparrow x_{k}^{z}, & \text { if } n=2 k \\ x_{k}^{y} \uparrow x_{k+1}^{z}, & \text { if } n=2 k+1\end{cases}$

Let $\bar{\varrho}$ be a metric on $\bar{W}$ induced by $\uparrow$. In the frame $(\bar{W}, \uparrow)$, a $\uparrow$-ball of radius $m$ and center $x \in W$ will be denoted by

$$
(x):=x \uparrow^{m}=\{x\} \cup\left\{x_{\ell}^{y} \mid x \Uparrow y, x \neq y, 1 \leqslant \ell<n\right\} .
$$

Obviously, $\bar{W}=\bigsqcup_{x \in W}(x)$. Now we establish some properties of $(\bar{W}, \uparrow)$ and $\bar{\varrho}$.

(1) $\forall x \in W \forall a, b \in(x) \quad \bar{\varrho}(a, b)<n$.

- By definition of $\uparrow$; its second item is essential here.

(2) $\forall x, y \in W . \quad x \Uparrow y \Rightarrow \forall a \in(x) \exists b \in(y) \quad a \uparrow^{n} b$.

- For $x=y$ this follows from (1). If $\varrho(x, y)=1$ then $b:=y_{m}^{x}$ suits for any $a \in(x)$, since $\bar{\varrho}\left(a, x_{m}^{y}\right)<n$ by $(1), \bar{\varrho}\left(x_{m}^{y}, b\right)=1$, and so $a \uparrow^{n} b$.

(3) $\forall x, y \in W . \quad \varrho(x, y)>1 \Rightarrow \forall a \in(x) \forall b \in(y) \quad \bar{\varrho}(a, b)>n$.

Assume that a minimal $\uparrow$-chain connecting $a$ and $b$ goes through a sequence of balls $(x)=\left(x_{0}\right),\left(x_{1}\right), \ldots,\left(x_{s}\right)=(y)$, where $x_{0} \Uparrow x_{1} \Uparrow \ldots \Uparrow x_{s}$. Since $\varrho(x, y)>1$, $s \geqslant 2$. Let $z:=x_{1}, t:=x_{2}$. Then this $\uparrow$-chain contains a subchain going through $x_{m}^{z}, z_{m}^{x}, z_{m}^{t}$, and $t_{m}^{z}$. Since $\bar{\varrho}\left(x_{m}^{z}, z_{m}^{x}\right)=1=\bar{\varrho}\left(z_{m}^{t}, t_{m}^{z}\right)$ and $\bar{\varrho}\left(z_{m}^{x}, z_{m}^{t}\right)=n-1$ (for the latter, the second item of the definition of $\uparrow$ is essential), the length of the whole $\uparrow$-chain $\bar{\varrho}(a, b) \geqslant 1+(n-1)+1>n$.

Finally, for all $x \in W, a \in(x)$ and $p \in$ Var, we put $a \Perp^{\prime} p$ iff $x \models p$ (i.e., any point of the ball $(x)$ validates the same variables as $x$ does).

Lemma 3.23 Any two points of a ball validate the same formulas of the form $F^{n}$ :

$$
\forall x \in W \forall a, b \in(x) \forall F \in \mathrm{Fm} \quad a \Perp^{\prime} F^{n} \Leftrightarrow b \|^{\prime} F^{n} .
$$


- By induction on $F$. The atomic and boolean cases are trivial. Now it is convenient to consider the case $F=\diamond G$; moreover, due to the symmetry of the claim, it suffices to prove only the ' $\Rightarrow$ ' implication.

$a \models^{\prime} F^{n}$, i.e., $a \models^{\prime} \diamond^{n} G^{n}$ iff $\exists a^{\prime} \downarrow^{n} a a^{\prime} \models^{\prime} G^{n}$. Take $y \in W$ such that $a^{\prime} \in(y)$. Since $\bar{\varrho}\left(a, a^{\prime}\right) \leqslant n$, by (3) we have $\varrho(x, y) \leqslant 1$, i.e., $x \Uparrow y$. By $(2)$, for our $b \in(x)$ there exists $b^{\prime} \in(y)$ such that $b \uparrow^{n} b^{\prime}$. As $a^{\prime}$ and $b^{\prime}$ are in the same ball (y), by I.H., $a^{\prime} \models^{\prime} G^{n}$ implies $b^{\prime} \models^{\prime} G^{n}$, whence $\left.b\right|^{\prime} \diamond^{n} G^{n}$, i.e., $b \models^{\prime} F^{n}$.

Lemma 3.24 (Main) $\forall x \in W \forall F \in \mathrm{Fm} \quad x \models F \Leftrightarrow x \models^{\prime} F^{n}$.

- By induction on $F$. Let $F=\square G$. We use the following obvious equality:

$$
\begin{aligned}
& x \uparrow^{n}=(x) \cup\left\{y_{m}^{x} \mid x \Uparrow y, x \neq y\right\} . \\
& x \models F \Leftrightarrow x \models \square G \Leftrightarrow \forall y \Downarrow x \quad y \models G \quad \Leftrightarrow \quad \text { (by I.H.) } \\
& \forall y \Downarrow x \quad y \models^{n} \Leftrightarrow\left(\begin{array}{l}
(\Leftrightarrow) \text { Lemma 3.23 } \\
(\Leftarrow) \text { in particular }
\end{array}\right) \\
& \forall y \Downarrow x \forall b \in(y) \quad b \models G^{n} \Leftrightarrow\left(\begin{array}{l}
(\Rightarrow) \text { in particular } \\
(\Leftarrow) \text { Lemma 3.23 }
\end{array}\right) \\
& \forall a \in(x) \quad a \Perp^{\prime} G^{n} \& \\
& \& \forall y \in x \Uparrow \backslash\{x\} \quad y_{m}^{x} \models^{\prime} G^{n} \Leftrightarrow \text { (by equality (b)) } \\
& \forall b \downarrow^{n} x \quad b \models^{\prime} G^{n} \Leftrightarrow x \Perp^{\prime} \square^{n} G^{n} \Leftrightarrow x \Perp^{\prime} F^{n} .
\end{aligned}
$$

Now, by the Main Lemma, $w \models A$ implies $w \models^{\prime} A^{n}$, Q.E.D.

Theorem $3.25 \varepsilon(\mathbf{B})=\alpha(\mathbf{B})=\infty$. Moreover, there exist infinitely many linear modalities which are non-analogous over $\mathbf{B}$.

Proof. Consider modalities $\nabla_{n}=\square^{n+1} \diamond^{n}$ and formulas $A_{m}=\square\left(p \rightarrow \square^{m} p\right)$. To prove the theorem we show that for all $n \geqslant 1$ and $m \geqslant 0$,

$$
\mathbf{B}\left(\nabla_{n}\right) \vdash A_{m} \Leftrightarrow m \leqslant n .
$$

$(\Leftarrow)$ Assume that $m \leqslant n$. We need $\mathbf{B}\left(\nabla_{n}\right) \vdash A_{m}$, i.e.,

$$
\mathbf{B} \vdash \square^{n+1} \diamond^{n}\left(p \rightarrow\left(\square^{n+1} \diamond^{n}\right)^{m} p\right) .
$$

As $\mathbf{B}$ has the rule (Nec), we put away the prefix $\square^{n+1}$. Furthermore, since $\mathbf{K} \vdash$ $\diamond(\varphi \rightarrow \psi) \leftrightarrow(\square \varphi \rightarrow \diamond \psi)$, it remains to prove in $\mathbf{B}$ a formula

$$
\square^{n} p \rightarrow \diamond^{n}\left(\square^{n+1} \diamond^{n}\right)^{m} p .
$$

Now, schematically, $\mathbf{B} \vdash \square^{n} \stackrel{\text { (ref) }}{\longrightarrow} \square^{m} \stackrel{\text { (sym) }}{\longrightarrow}\left(\square \square^{n} \diamond^{n}\right)^{m} \stackrel{(\text { ref })}{\longrightarrow} \diamond^{n}\left(\square \square^{n} \diamond^{n}\right)^{m}$, where the steps labelled by (ref) and (sym) use (zero or more times) the reflexivity and symmetry axioms, respectively.

$(\Rightarrow)$ Assume that $m>n$. Since the rule $(\mathrm{Nec})$ is reversible in $\mathbf{B}$ (due to the reflexivity axiom), we consider again the formula $(\sharp)$. To prove that $\mathbf{B} \nvdash(\sharp)$, we show that the reflexive symmetric chain shown in Figure 4 falsifies $(\sharp)$ at the point $x_{0}$ (in the Figure 4 the valuation of $p$ is also shown). 


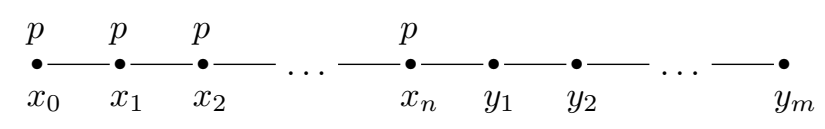

Figure 4: $x_{0} \not=(\sharp)$.

As $x_{0} \uparrow^{n}=\left\{x_{0}, \ldots, x_{n}\right\}, x_{0} \models \square^{n} p$. Now we prove $x_{0} \models \square^{n}\left(\diamond^{n+1} \square^{n}\right)^{m} \neg p$.

$$
\begin{aligned}
& \left.\forall a_{0} \downarrow^{n} x_{0} \exists b_{1} \downarrow^{n+1} a_{0} \text { (namely, } b_{1}:=y_{1}\right) \quad \forall a_{1} \downarrow^{n} b_{1} \\
& \exists b_{2} \downarrow^{n+1} a_{1} \text { (namely, } b_{2}:=y_{2} \text { ) } \forall a_{2} \downarrow^{n} b_{2} \\
& \text { : } \\
& \left.\exists b_{m} \downarrow^{n+1} a_{m-1} \text { (namely, } b_{m}:=y_{m}\right) \forall a_{m} \downarrow^{n} b_{m} \quad a_{m} \not \neq p,
\end{aligned}
$$

since $b_{m} \uparrow^{n}=y_{m} \uparrow^{n}=\left\{y_{m}, y_{m-1}, \ldots, y_{m-n}\right\}$ and $m-n \geqslant 1$.

\subsection{Expressibility of provability logics}

All definitions and facts concerning provability logics can be found in [2] or in the survey paper [7]. We recall some of them briefly.

Let $\mathcal{T}$ and $\mathcal{U}$ be two arithmetical theories, $\mathcal{T}$ recursively enumerable. Intuitively, the provability logic of $\mathcal{T}$ relative to $\mathcal{U}$ expresses those principles of provability in $\mathcal{T}$ that can be verified by means of $\mathcal{U}$. More precisely, consider an arithmetical interpretation of modal formulas which assign to each propositional variable an arithmetical sentence, respects boolean connectives, and translates $\square$ into a formula of provability in $\mathcal{T}$. Then the provability logic of $\mathcal{T}$ relative to $\mathcal{U}$ is the set of all modal formulas whose all interpretations of this kind are provable in $\mathcal{U}$. Every provability logic contains GL and is closed under (MP) and (Sub), but not necessarily under (RE) (hence not all of them are normal).

The basic provability logics are GL and the following two:

$\mathbf{D}=\mathbf{G L}\{\neg \square \perp, \square(\square p \vee \square q) \rightarrow(\square p \vee \square q)\}$ — the Dzhaparidze logic;

$\mathbf{S}=\mathbf{G L}\{\square p \rightarrow p\}$ - the Solovay logic.

Denote by $F_{n}$ the formula $\square^{n+1} \perp \rightarrow \square^{n} \perp$, for $n \in \omega=\{0,1, \ldots\}$. The Classification Theorem proved by L. D. Beklemishev (cf. [2]) states that the provability logics are exhausted by the following four families (here $\alpha, \beta \subseteq \omega, \beta$ cofinite):

$$
\begin{array}{ll}
\mathbf{G L}_{\alpha}=\mathbf{G L}\left\{F_{n} \mid n \in \alpha\right\}, & \mathbf{D}_{\beta}=\mathbf{D} \cap \mathbf{G L}_{\beta}^{-}, \\
\mathbf{G L}_{\beta}^{-}=\mathbf{G L}\left\{\bigvee_{n \notin \beta} \neg F_{n}\right\}, & \mathbf{S}_{\beta}=\mathbf{S} \cap \mathbf{G L}_{\beta}^{-} .
\end{array}
$$

The inclusion of logics within each family reflects the inclusion of their indices (i.e., $\alpha$ and $\beta$ ), whereas for any cofinite $\beta \subseteq \omega$ the following additional strict inclusions hold:

$$
\mathbf{G L}_{\beta} \subset \mathbf{D}_{\beta} \subset \mathbf{S}_{\beta} \subset \mathbf{G L}_{\beta}^{-} .
$$

The only provability logics closed under (RE) (and hence normal) are $\mathbf{G L}$ and $\mathbf{G L}_{n}^{-}:=$ $\mathbf{G L}_{[n, \infty)}^{-}=\mathbf{G L}\left\{\square^{n} \perp\right\}, n \geqslant 0$. From the results stated in [1] it follows that only the 
following provability logics are iterative:

$$
\begin{aligned}
\mathbf{G L} \subset \mathbf{G L}_{[1, \infty)} & \subset \mathbf{D}_{[1, \infty)} \subset \mathbf{S}_{[1, \infty)} \subset \mathbf{G L}_{[1, \infty)}^{-} \\
\cap & \cap \\
\mathbf{G L}_{\omega} & \subset \mathbf{D}_{\omega}=\mathbf{D} \subset \mathbf{S}_{\omega}=\mathbf{S} \subset \mathbf{G L}_{\omega}^{-}=\mathrm{Fm}
\end{aligned}
$$

If all formulas of a modal logic $L$ are true under all arithmetical interpretations in the standard model of arithmetic then $L$ is called regular, otherwise singular. Logics $\mathbf{G L}_{\alpha}, \mathbf{D}_{\beta}$, and $\mathbf{S}_{\beta}$ are regular and $\mathbf{S}$ is the greatest of them, whereas $\mathbf{G} \mathbf{L}_{\beta}^{-}$are singular. As we shall see, values of $\varepsilon(L)$ and $\alpha(L)$ are infinite for any regular provability logic and finite for any singular one.

Theorem 3.26 $\varepsilon(L)=\alpha(L)=\infty$, for any logic $L \in[\mathbf{K}, \mathbf{S}]$.

Proof. We use the following completeness theorem for $\mathbf{S}$ proved by A. Visser (cf. [2, $7]): \mathbf{S} \vdash A$ iff $A$ is true in all tail-models. We shall not bore the reader by giving the definition of a tail-model; for our purposes it will be enough to know that any irreflexive $(\omega+1)^{*}$-type linearly ordered Kripke model $(W, \prec, \mid=)$ such that, for some $r \in W$, a valuation of any variable is the same at all points of $\{x \mid x \prec r\}$ is a tailmodel, and that a formula is said to be true in a tail-model if it is true at its least point.

Take $X$ to be the set of odd natural numbers and $n_{k}:=2 k$. First we prove that modalities $\nabla_{k}:=\nabla_{n_{k}}^{X}, k \geqslant 1$, are non-equivalent in $\mathbf{S}$ (this will imply $\varepsilon(\mathbf{S})=\infty$ and hence $\varepsilon(L)=\infty)$. Consider a tail-model $(W,<, \models)$, where $W=\{b\} \cup V, V=\{i \in \mathbb{Z} \mid$ $i \leqslant 2 k\}$, the restriction of $<$ to $V$ is the ordinary 'less-than' relation, $b<i$ whenever $i \in V$, and for all $x \in W, x \mid=p$ iff $x \leqslant 0$ or $x$ is odd. Then $b \not \nabla_{k} p \rightarrow \nabla_{k+1} p$.

Now we prove an analog of Lemma 3.15. Put $n:=\left|X \cap\left(0, n_{k}\right]\right|=k$.

Lemma 3.27 If $m<n$ then $\mathbf{S}\left(\nabla_{k}\right) \forall A_{N}^{m}$, for any $N \geqslant n$.

- Take a frame $(W,<)$ as above and the following valuation $\models$ of $p_{1}, \ldots, p_{N}$ : for all $x \in W$, if $x \leqslant 0$ then $\left.x\right|^{\prime} p_{1}$ else $\left.x\right|^{\prime} p_{j}$ iff $x=2 j-1$, for all $j, 1 \leqslant j \leqslant n$. It is not hard to see that $\left.b\right|^{\prime} \neq \operatorname{tr}_{\nabla_{k}}\left(A_{N}^{m}\right)$.

The theorem follows from Lemmas 3.14 and 3.27.

Theorem $3.28 \varepsilon\left(\mathbf{G L}_{\beta}^{-}\right)<\infty$ and $\alpha\left(\mathbf{G L}_{\beta}^{-}\right)<\infty$, for any cofinite $\beta \subseteq \omega$.

Proof. First we consider the logics $\mathbf{G L}_{n}^{-}, n \geqslant 0$. Since they are closed under (RE), it suffices to show that $\varepsilon\left(\mathbf{G L}_{n}^{-}\right)<\infty$.

A subformula $F$ of a formula $A$ is said to be on the depth $n$ if it is in the scope of exactly $n \square$ s. Let $A^{(n)}$ be the result of substituting of $\perp$ for all subformulas of $A$ that are on the depth $n$. If a modality $\nabla$ is induced by a formula $\varphi$ then denote by $\nabla^{(n)}$ a modality induced by $\varphi^{(n)}$. The notion of degree of a formula is defined as usual; the degree of a modality is the degree of the corresponding formula.

We claim that $\mathbf{G L}_{n}^{-} \vdash A \leftrightarrow A^{(n)}$, for all $A \in \mathrm{Fm}$; this follows from

$$
\mathbf{K} \vdash \square^{n} \perp \rightarrow\left(A \leftrightarrow A^{(n)}\right),
$$


which is easily proved by induction on $n$. Hence every modality $\nabla$ is equivalent in $\mathbf{G L}_{n}^{-}$to a modality $\nabla^{(n)}$ of degree non-larger than $n$. But (even in $\mathbf{K}$ ) there exists only a finite number of non-equivalent modalities of bounded degree. Thus $\varepsilon\left(\mathbf{G L}_{n}^{-}\right)<\infty$.

Now consider $L:=\mathbf{G L}_{\beta}^{-}$for an arbitrary cofinite $\beta \subseteq \omega$. Then $[n, \infty) \subseteq \beta$ for some $n \geqslant 0$, hence $\mathbf{G L}_{n}^{-} \subseteq L$. So $\varepsilon(L) \leqslant \varepsilon\left(\mathbf{G L}_{n}^{-}\right)<\infty$, by antimonotonicity of $\varepsilon(\cdot)$, and $\alpha(L) \leqslant \varepsilon\left(\mathbf{G L}_{n}^{-}\right)<\infty$, by Lemma 3.1 .

\section{Embeddings of logics}

In this section we are mainly focused on obtaining results stating that some particular logics are not embeddable into some others. These results are of two sorts. The first ones are based on the simple observation that if a logic $L$ is richer, in a sense, than a logic $M$ then $L$ is not embeddable into $M$. The second ones involve an unexpected fact that the presence of the symmetry axiom, i.e. the formula $p \rightarrow \square \diamond p$, in a logic prevents this logic from being embeddable into some strong logics, namely, into modalised logics (see Definition 4.4 below). A surprising corollary is, for example, that the "quite simple" logic $\mathbf{S 5}$ having $\varepsilon(\mathbf{S 5})=\alpha(\mathbf{S 5})=16$ is not embeddable into "rich enough" logics such as $\mathbf{K}$.

In the sequel, $L$ and $M$ range over logics and $\Upsilon=\{\perp, \bigcirc, \neg, \top\}$.

Lemma 4.1 If $L \hookrightarrow M$ then the following conditions hold:

(1) $\varepsilon(L) \leqslant \varepsilon(M)$ and $\alpha(L) \leqslant \alpha(M)$;

(2) if $M$ is closed under (RE) then so is $L$;

(3) if $L$ has a proper constant then so does $M$;

(4) the number of non-equivalent constants in $L$ is no more than in $M$;

(5) if $M \subseteq \boldsymbol{\Lambda}_{\nabla}$ for some $\nabla \in \Upsilon$ then $L \subseteq \boldsymbol{\Lambda}_{\nabla^{\prime}}$ for some $\nabla^{\prime} \in \Upsilon$.

Proof. Items (1-4) are trivial. In (5), if $L=M(\Delta)$ then $M \subseteq \boldsymbol{\Lambda}_{\nabla}$ implies $L=$ $M(\Delta) \subseteq \boldsymbol{\Lambda}_{\nabla}(\Delta)=\boldsymbol{\Lambda}_{\nabla^{\prime}}$ for $\nabla^{\prime}:=\operatorname{tr}_{\nabla}(\Delta) \in \Upsilon$.

Before giving a corollary, we recall the notion of the trace of a logic (cf. [2]).

Definition 4.2 If $(W, \uparrow)$ is a finite irreflexive transitive (f.i.t.) tree then the depth $d(x)$ of an element $x \in W$ is defined as follows: if $x$ is a leaf then $d(x):=0$, else $d(x):=1+\max \{d(y) \mid x \uparrow y\}$. The height of a tree is the depth of its root. The trace of a formula is the set $t(A)$ of heights of all f.i.t. trees falsifying $A$ at their roots. The trace of a logic $L$ is $t(L):=\bigcup_{A \in L} t(A)$.

It is worth noting that $t\left(\mathbf{G L}_{\alpha}\right)=\alpha$ and $t\left(\mathbf{D}_{\beta}\right)=t\left(\mathbf{S}_{\beta}\right)=t\left(\mathbf{G L}_{\beta}^{-}\right)=\beta$.

Corollary 4.3 $L \nrightarrow M$ in any of the following cases:

(1) $L \in[\mathbf{K}, \mathbf{G r z}] \cup[\mathbf{K}, \mathbf{S}]$ and $M \supseteq \mathbf{K} \mathbf{5}$;

(2) $L \subseteq \mathbf{G L}$ or $L \subseteq \mathbf{K 5}$ or $L \subseteq \mathbf{K B}, M$ is normal, and $M \vdash \neg \square \perp$;

(3) $L$ is a provability logic other than $\mathbf{G L}$ or $\mathbf{G L}_{n}^{-}$and $M$ is normal;

(4) $L$ is a regular provability logic and $M$ is a singular one;

(5) $L \subseteq \mathbf{G L}$ and $M \supseteq \mathbf{G L}_{\alpha}$ for some cofinite $\alpha \subseteq \omega$;

(6) $L, M \supseteq \mathbf{G L}$ and $0 \in t(L) \backslash t(M)$;

(7) $\mathbf{G L} \subseteq L \nsubseteq \boldsymbol{\Lambda}_{\top}$ and $M$ is normal. 
Proof. (1), (4) Here $\varepsilon(L)=\infty$ but $\varepsilon(M)<\infty$ by the results of Subsection 3.2.

(2) The constant $\square \perp$ is proper in $L$ but any constant is trivial in $M$.

(3) $M$ is closed under (RE) whereas $L$ is not.

(5) It is known (see [3, Chapter 7]) that any constant is equivalent in GL to a truth-functional compound of $\square^{n} \perp, n \geqslant 0$, but since $M \vdash \square^{n} \perp \leftrightarrow \square^{n+1} \perp$, for all $n \in \alpha, M$ has only finite number of non-equivalent constants. On the other hand, the constants $\square^{n} \perp$ are non-equivalent in $L$.

(6) For any logic $N \supseteq \mathbf{G L}$ : firstly, $N$ is not contained in $\boldsymbol{\Lambda}_{\perp}, \boldsymbol{\Lambda}_{\bigcirc}$, and $\boldsymbol{\Lambda}_{\neg}$; secondly, $N \subseteq \boldsymbol{\Lambda}_{\top}$ iff all formulas of $N$ are true in all f.i.t. trees of height 0 , iff $0 \notin t(N)$. Hence $M \subseteq \boldsymbol{\Lambda}_{\top}$ but $L \nsubseteq \boldsymbol{\Lambda}_{\nabla}$, for any $\nabla \in \Upsilon$.

(7) As in (6), LI $\boldsymbol{\Lambda}_{\nabla}$, for any $\nabla \in \Upsilon$. But in [10] it was shown that, for any normal logic $M$, if $M \vdash \neg \square \perp$ then $M \subseteq \boldsymbol{\Lambda}_{\bigcirc}$ else $M \subseteq \boldsymbol{\Lambda}_{\top}$.

Definition 4.4 A formula $A$ is modalised in $p$ if every occurrence of the variable $p$ is in the scope of $\square$. In particular, if $p$ does not occur in $A$ then $A$ is modalised in $p$. $A$ is called modalised if it is modalised in every variable; in other words, if $A$ is a truth-functional compound of formulas of the form $\square F$.

If $\vec{p}=\left(p_{1}, \ldots, p_{n}\right)$ is the list of all variables in that $A$ is not modalised then $A$ is truth-functionally equivalent to a decomposition w.r.t. $\vec{p}$ of the form

$$
A \longleftrightarrow \bigvee_{\vec{\sigma} \in \mathbf{2}^{n}}\left(\vec{p}^{\vec{\sigma}} \wedge B_{\vec{\sigma}}\right),
$$

where $B_{\vec{\sigma}}$ are modalised formulas.

A logic $M$ is modalised if, for all $A \in \mathrm{Fm}, M \vdash A$ implies $M \vdash B_{\vec{\sigma}}$, for all $\vec{\sigma} \in \mathbf{2}^{n}$, where $B_{\vec{\sigma}}$ are taken from the decomposition $(\star)$ of $A$. To put it in another way, $M$ is modalised if it does not prove any nontrivial truth-functional combination of variables and modalised formulas.

Lemma 4.5 The logics $\mathbf{K}, \mathbf{K} 4, \mathbf{K 5}, \mathbf{K} 45$, and $\mathbf{G L}$ are modalised.

Proof. Consider GL first. Take the decomposition $(\star)$ of a formula $A$ and assume that $\mathbf{G L} \forall \forall B_{\vec{\sigma}}$ for some $\vec{\sigma} \in \mathbf{2}^{n}$. Then there exists a f.i.t. tree with a root $r$ such that $r \not \neq B_{\vec{\sigma}}$. Since $r$ is inaccessible from any point of the tree, the condition $r \not \mid B_{\vec{\sigma}}$ is independent of a valuation of variables at $r$, so we change $\models$ by putting $r \models p_{i}$ iff $\sigma_{i}=\mathrm{T}$. Then $r \not \neq A$ and so GL $\not \forall A$.

To apply the same argument to $L \in\{\mathbf{K}, \mathbf{K} 4, \mathbf{K 5}, \mathbf{K} 45\}$, we only need to prove the following: If $L \forall \forall A$ then there exists an $L$-frame $(W, \uparrow)$, a valuation $\models$, and an element $r \in W$ such that $r \not \neq A$ and $\forall x \in W \neg(x \uparrow r)$.

But this is simple: By completeness of $L$, if $L \forall B$ then there is an $L$-frame $(W, \uparrow)$, a valuation $=$ and an element $r \in W$ such that $r \not \neq B$. Now add to $W$ a new element $\bar{r}$, thus obtaining $\bar{W}:=W \cup\{\bar{r}\}$, put $\Uparrow:=\uparrow \cup\{\langle\bar{r}, x\rangle \mid r \uparrow x\}$, and extend $=$ to $\bar{r}$ by putting $\bar{r} \models p$ iff $r \models p$, for all $p \in$ Var. In each of our four cases, $(\bar{W}, \Uparrow)$ is an $L$-frame, $\forall x \in \bar{W} \neg(x \Uparrow \bar{r})$, and $\bar{r} \models F$ iff $r \models F$, for all $F \in$ Fm. Thus, $\bar{r} \not \neq A$.

Lemma 4.6 If $L$ is a modalised logic and $X$ is a set of modalised formulas then $L X$ is a modalised logic. 
Proof. If $L X \vdash A$ then $L \vdash \bigwedge \Gamma \rightarrow A$ for some finite set $\Gamma$ of substitution instances of formulas in $X$. Since formulas in $\Gamma$ are modalised, from the decomposition $(\star)$ of the formula $A$ we obtain

$$
(\bigwedge \Gamma \rightarrow A) \longleftrightarrow \bigvee_{\vec{\sigma} \in \mathbf{2}^{n}}\left(\vec{p}^{\vec{\sigma}} \wedge\left(\bigwedge \Gamma \rightarrow B_{\vec{\sigma}}\right)\right),
$$

hence $L \vdash \bigwedge \Gamma \rightarrow B_{\vec{\sigma}}$, for all $\vec{\sigma} \in \mathbf{2}^{n}$, and finally $L X \vdash B_{\vec{\sigma}}$.

Corollary 4.7 $\mathbf{G L}_{\alpha}, \mathbf{D}_{\beta}$, and $\mathbf{G L}_{\beta}^{-}$are modalised, for any $\alpha, \beta \subseteq \omega, \beta$ cofinite.

Theorem 4.8 Suppose a logic $L \supseteq \mathbf{K B}$ is normal, a logic $M \supseteq \mathbf{E}$ is modalised, and $L \hookrightarrow M$. Then $L \supseteq \boldsymbol{\Lambda}_{\bigcirc \top}$.

Proof. We prove a bit more: if $L \subseteq M(\nabla)$ for some $\nabla$ then $\boldsymbol{\Lambda}_{\bigcirc \top} \subseteq M(\nabla)$.

Let the decomposition $(\star)$ of the formula $\nabla p$ be

$$
\nabla p \longleftrightarrow\left[(p \wedge \Delta p) \vee\left(\neg p \wedge \Delta^{\prime} p\right)\right],
$$

where the formulas $\Delta p$ and $\Delta^{\prime} p$ are modalised.

Since $L$ contains the distributivity axiom, $M \vdash \nabla(p \rightarrow q) \rightarrow(\nabla p \rightarrow \nabla q)$. The decomposition $(\star)$ splits this into the four conditions (we omit ' $M \vdash$ '):

$$
\begin{aligned}
& \text { (a) } \Delta(p \rightarrow q) \rightarrow\left(\Delta^{\prime} p \rightarrow \Delta^{\prime} q\right) ; \\
& \text { (b) } \Delta(p \rightarrow q) \rightarrow\left(\Delta^{\prime} p \rightarrow \Delta q\right) ; \\
& \text { (c) } \Delta^{\prime}(p \rightarrow q) \rightarrow\left(\Delta p \rightarrow \Delta^{\prime} q\right) ; \\
& \text { (d) } \Delta(p \rightarrow q) \rightarrow(\Delta p \rightarrow \Delta q)
\end{aligned}
$$

Further, the decomposition $(\star)$ applied to the $\nabla$-translation of the symmetry axiom $(A B)$ yields the only one condition (since the other one is a tautology):

$$
\left(\neg \Delta^{\prime} \neg p \wedge \Delta \neg \nabla \neg p\right) \vee\left(\Delta^{\prime} \neg p \wedge \Delta^{\prime} \neg \nabla \neg p\right),
$$

which is truth-functionally equivalent to a conjunction of the following two conditions (we replaced $\neg p$ by $p$; this is correct, for we could first substitute $\neg p$ for $p$ and then, equivalently even in $\mathbf{E}$, replace $\neg \neg p$ by $p$ ):

$$
\begin{aligned}
& \text { (e) } \Delta^{\prime} p \rightarrow \Delta^{\prime} \neg \nabla p \text {; } \\
& \text { (f) } \Delta^{\prime} p \vee \Delta \neg \nabla p .
\end{aligned}
$$

Finally, $L$ is closed under (Nec), hence the set of $\nabla$-translations of theorems of $L$ is closed in $M$ under the rule

$$
\text { (g) } A \vdash \Delta A \text {. }
$$

Now, since $M \vdash \Delta(p \rightarrow p)$, substituting $p$ for $q$ in (b) yields

(h) $\Delta^{\prime} p \rightarrow \Delta p$. By (e), from (f) it follows that

(i) $\Delta^{\prime} \neg \nabla p \vee \Delta \neg \nabla p$, whence by the scheme (h), we have

(j) $\Delta \neg \nabla p$; by the decomposition ( $\star$ ) of $\nabla p$, it is equivalent (even in $\mathbf{E}$ ) to

(k) $\Delta\left[(\Delta p \rightarrow \neg p) \wedge\left(\Delta^{\prime} p \rightarrow p\right)\right]$. Now (d) and (g) imply that $L(\Delta)$ is a normal logic, 
hence we have a principle of monotonicity:

(l) $\Delta(r \wedge s) \rightarrow \Delta r$, which applied to (k), due to (Sub), yields

(m) $\Delta(\Delta p \rightarrow \neg p)$, whence by the distributivity (d), we have

(n) $\Delta \Delta p \rightarrow \Delta \neg p$. From (m) we infer by the rule (g), that

(o) $\Delta \Delta(\Delta p \rightarrow \neg p)$; we apply the scheme (n) to this and get

(p) $\Delta(p \wedge \Delta p)$; by monotonicity (l), we obtain finally

(q) $\Delta p$. Thus, the decomposition $(\star)$ of $\nabla p$ turns into

(r) $\nabla p \leftrightarrow\left(p \vee \Delta^{\prime} p\right)$, in particular,

(s) $\nabla \perp \leftrightarrow \Delta^{\prime} \perp$. Now by (q), from (a) we have $\Delta^{\prime} p \rightarrow \Delta^{\prime} q$, hence

(t) $\Delta^{\prime} p \leftrightarrow \Delta^{\prime} \perp$. From (s) and (t) we have $\Delta^{\prime} p \leftrightarrow \nabla \perp$, therefore (r) turns into

(u) $\nabla p \leftrightarrow(p \vee \nabla \perp)$. By the axiomatics of $\boldsymbol{\Lambda}_{\bigcirc \top}$ (see Theorem 2.5), we have proved the required inclusion: $\boldsymbol{\Lambda}_{\bigcirc \top} \subseteq M(\nabla)$.

Before deriving corollaries, note that if $L \supset \boldsymbol{\Lambda}_{\bigcirc \top}$ then either $L=\boldsymbol{\Lambda}_{\bigcirc}=$ Triv or $L=\boldsymbol{\Lambda}_{\top}=$ Ver. Indeed, $L \vdash \square p \leftrightarrow p \vee \square \perp$ and hence $L$ is a prime logic (since it is the logic of the modality $\square$ equivalent to the prime modality $p \vee \square \perp$ ), but $\boldsymbol{\Lambda}_{\bigcirc}$ and $\boldsymbol{\Lambda}_{\top}$ are the only prime logics strictly containing $\boldsymbol{\Lambda}_{\bigcirc \top}$.

Now we prove an auxiliary lemma.

Lemma 4.9 Suppose a normal logic $L$ is given by a set of axioms and the rules (MP), (Sub), and (Nec), and $L(\square) \supseteq L$. Then $L(\square)=L+\{\square p \rightarrow p\}$.

Proof. The inclusion ' $\supseteq$ ' is obvious. To see that $L(\square) \subseteq L_{1}:=L+\{\square p \rightarrow p\}$ observe that $L_{1} \vdash \square p \leftrightarrow \square p$, hence $L_{1} \vdash A \leftrightarrow \operatorname{tr}_{\square}(A)$, for all $A \in \mathrm{Fm}$. So, if $A \in L(\square)$, i.e., $\operatorname{tr}_{\varpi}(A) \in L \subseteq L_{1}$ then $A \in L_{1}$.

It is known (cf. [3, Chapter 12]) that $\mathbf{G L}(\odot)=\mathbf{G r z}$. From Lemma 4.9 we conclude: $\mathbf{K}(\sqcup)=\mathbf{T}, \mathbf{K} \mathbf{4}(\odot)=\mathbf{S} \mathbf{4}$, and $\mathbf{K B}(\odot)=\mathbf{B}$.

Theorem 4.10 Suppose $L$ is a normal logic containing $\mathbf{K B}$ (e.g., any extension of S5) and different from Triv, Ver, and Triv $\cap$ Ver; $M$ is one of the following logics: $\mathbf{K}, \mathbf{K} 4, \mathbf{K 5}, \mathbf{K} 45, \mathbf{T}, \mathbf{S 4}, \mathbf{G r z}, \mathbf{G L}_{\alpha}, \mathbf{D}_{\beta}, \mathbf{G L}_{\beta}^{-}(\alpha, \beta \subseteq \omega, \beta$ cofinite). Then $L \nrightarrow M$.

Proof. That all extensions of S5 are normal is shown in [16]. Insofar as the logics $\mathbf{K}, \mathbf{K 4}, \mathbf{K 5}, \mathbf{K} 45, \mathbf{G L}_{\alpha}, \mathbf{D}_{\beta}$, and $\mathbf{G L}_{\beta}^{-}$are modalised (by Lemmas 4.5 and 4.6), for them the claim follows from Theorem 4.8. For $\mathbf{T}, \mathbf{S} 4$, and $\mathbf{G r z}$ this follows from $\mathbf{T} \hookrightarrow \mathbf{K}, \mathbf{S} \mathbf{4} \hookrightarrow \mathbf{K} \mathbf{4}, \mathbf{G r z} \hookrightarrow \mathbf{G L}$, and the transitivity of ' $\hookrightarrow$ '.

We conclude with a positive result.

Theorem 4.11 GL $\hookrightarrow \mathbf{G L}_{\alpha}$, for any finite $\alpha \subseteq \omega$.

Proof. In [1] GL is stated to be iterative: $\mathbf{G L}\left(\square^{n}\right)=\mathbf{G L}$, for all $n \geqslant 1$. Take $n \geqslant 1$ such that $\alpha \subseteq[0, n)$ and consider two cases. Recall that $A^{n}=\operatorname{tr}_{\square^{n}}(A)$.

Case $0 \notin \alpha$. We claim that $\mathbf{G L}_{\alpha}\left(\square^{n}\right)=\mathbf{G L}\left(\square^{n}\right)$. The inclusion ' $\supseteq$ ' is clear. Now assume that $A \in \mathbf{G L}_{\alpha}\left(\square^{n}\right)$, i.e., $\mathbf{G L}_{\alpha} \vdash A^{n}$. Then the trace $t\left(A^{n}\right) \subseteq \alpha \subseteq[0, n)$. So $A^{n}$ could be falsified only at the root of some f.i.t. tree of height less than $n$. Obviously, $A^{n}$ is true at a point of depth less than $n$ iff $\operatorname{tr}_{\top}(A)$ is. But since $0 \notin \alpha$, 
$\mathbf{G L}_{\alpha} \subseteq \boldsymbol{\Lambda}_{\top}$, so $\operatorname{tr}_{\top}(A)$ is a tautology and is true in any tree, hence so is $A^{n}$. Thus $\mathbf{G L} \vdash A^{n}$ and $A \in \mathbf{G L}\left(\square^{n}\right)$.

Case $0 \in \alpha$. We argue that $\mathbf{G L}_{\alpha}\left(\square^{n+1}\right)=\mathbf{G L}\left(\square^{n+1}\right)$. Again, ' $\supseteq$ ' is obvious. Put $m:=n+1$ and $F_{\alpha}:=\bigwedge_{n \in \alpha} F_{n}$. Clearly, $\mathbf{G L}_{\alpha} \vdash A$ iff $\mathbf{G L} \vdash F_{\alpha} \rightarrow A$. Assume that $A \notin \mathbf{G L}\left(\square^{m}\right)$, then there exists a f.i.t. tree $(W, \uparrow, \models)$ with the root $r$ such that $r \mid \neq A^{m}$.

If $d(r)<n$ then we add to this tree a chain $x_{n} \uparrow x_{n-1} \uparrow \ldots \uparrow x_{d(r)}:=r$ (so that $\left.d\left(x_{n}\right)=n\right)$ and extend $\models$ so that $x_{n}$ and $r$ validate the same variables. Then it is readily seen that $x_{n}$ and $r$ validate the same formulas of the form $F^{m}$, in particular, $x_{n} \not \neq A^{m}$.

So, without loss of generality we can assume that $d(r) \geqslant n$. Then $r \models F_{\alpha}$, since $t\left(F_{\alpha}\right)=\alpha \subseteq[0, n)$. Hence $r \not \neq F_{\alpha} \rightarrow A^{m}$, GL $\forall F_{\alpha} \rightarrow A^{m}, \mathbf{G L}_{\alpha} \not \forall A^{m}$, and finally $A \notin \mathbf{G L}_{\alpha}\left(\square^{m}\right)$.

\section{Acknowledgements}

The author is grateful to E. Yu. Nogina for the supervision during the research, to T. L. Sidon who carefully read the text and suggested several significant improvements in presentation, and to V.B. Shehtman for helpful comments.

\section{References}

[1] M. Abashidze, Property of iterativity in provability logics, Proc. of the 8th AllUnion Conf. on Math. Logic, Moscow, September 1986, p. 4. (Russian).

[2] L. D. Beklemishev, Classification of Propositional Provability Logics. Amer. Math. Soc. Transl. (2), 192:1-56.

[3] G. Boolos, Logic of Provability. Cambridge University Press, 1993.

[4] A. Chagrov, M. Zakharyaschev, Modal Logic. Oxford Science Publications, 1997.

[5] R. Feys, Modal Logic. Gauthier-Villas, Paris, 1965.

[6] I. L. Humberstone, The logic of non-contingency, Notre Dame Journal of Formal Logic, 1995, 36(2):214-229.

[7] D. de Jongh and G. Japaridze, The logic of provability. Handbook of Proof Theory (S. R. Buss, ed.), Studies in Logic and the Foundations of Mathematics. Elsevier, Amsterdam, 1998, vol. 137, 475-546.

[8] M. Kracht and F. Wolter, Normal monomodal logics can simulate all others, to appear in The Journal of Symbolic Logic.

[9] S. T. Kuhn, Minimal non-contingency logic, Notre Dame Journal of Formal Logic, 1995, 36(2):230-234.

[10] D. Makinson, Some embedding theorems for modal logic, Notre Dame Journal of Formal Logic, 1971, 12(2):252-254. 
[11] D. Makinson, There are infinitely many Diodorean modal functions, The Journal of Symbolic Logic, 1966, 31(4):406-408.

[12] H. Montgomery, R. Routley, Contingency and non-contingency bases for normal modal logics, Logique et analyse, 9 (1966), 318-328.

[13] H. Montgomery, R. Routley, Non-contingency axioms for $S 4$ and S5, Logique et analyse, 11 (1968), 422-424.

[14] H. Montgomery, R. Routley, Modalities is a sequence of normal non-contingency modal systems, Logique et analyse, 12 (1969), 225-227.

[15] V. Routley, There are infinitely many modalities in S6, 42 in S7, 18 in S8, and 14 in S8.5, The Journal of Symbolic Logic, 1969.

[16] S. J. Scroggs, Extensions of the Lewis system S5, The Journal of Symbolic Logic, 1951, 16(2):112-120.

[17] T. Sugihara, The number of modalities in $T$ supplemented by the axiom $C L^{2} p L^{3} p$, The Journal of Symbolic Logic, 1962, 27(4):407-408. 